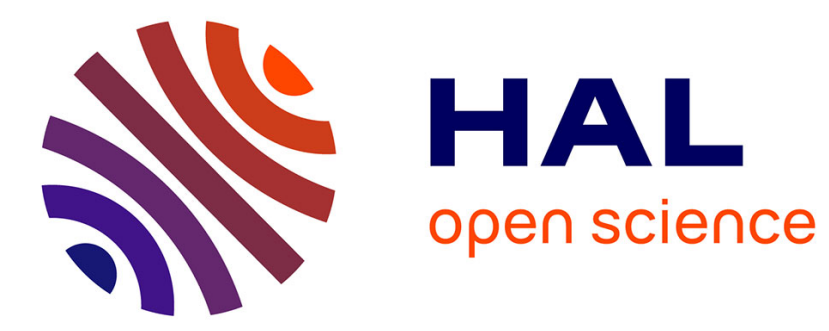

\title{
Population balance modelling of bubble columns under the heterogeneous flow regime
}

Luca Gemello, Cécile Plais, Frédéric Augier, Daniele L. Marchisio

\section{To cite this version:}

Luca Gemello, Cécile Plais, Frédéric Augier, Daniele L. Marchisio. Population balance modelling of bubble columns under the heterogeneous flow regime. Chemical Engineering Journal, 2019, 372, pp.590-604. 10.1016/j.cej.2019.04.109 . hal-02179205

\section{HAL Id: hal-02179205 \\ https://hal-ifp.archives-ouvertes.fr/hal-02179205}

Submitted on 10 Jul 2019

HAL is a multi-disciplinary open access archive for the deposit and dissemination of scientific research documents, whether they are published or not. The documents may come from teaching and research institutions in France or abroad, or from public or private research centers.
L'archive ouverte pluridisciplinaire HAL, est destinée au dépôt et à la diffusion de documents scientifiques de niveau recherche, publiés ou non, émanant des établissements d'enseignement et de recherche français ou étrangers, des laboratoires publics ou privés. 


\title{
Population balance modelling of bubble columns under the heterogeneous flow regime
}

\author{
Luca Gemello ${ }^{\mathrm{a}, \mathrm{b}, *}$, Cécile Plais ${ }^{\mathrm{a}}$, Frédéric Augier ${ }^{\mathrm{a}}$, Daniele L. Marchisio ${ }^{\mathrm{b}}$ \\ ${ }^{a}$ IFP Energies nouvelles, Rond-point de l'échangeur de Solaize, 69360 Solaize (69), \\ France \\ ${ }^{b}$ Dipartimento di Scienza Applicata e Tecnologia, Istituto di Ingegneria Chimica, \\ Politecnico di Torino, Corso Duca degli Abruzzi 24, 10129 Torino (TO), Italy
}

\begin{abstract}
Industrial bubble columns generally operate under the so-called heterogeneous regime and with high gas volume fractions, implying a fundamental role of the coalescence and breakage phenomena. These columns are usually simulated thanks to Eulerian CFD modelling, whereas the local bubble size distribution can be estimated with population balance models. The coupling can be realized with the Quadrature Method of Moments in order to minimize the CPU time consumption. In this work, the CFD-PBM/QMOM approach is validated using experimental data. Various qualities of water and two spargers are investigated. Considering different additives and spargers allow studying separately the effect of breakage and coalescence. In particular, a perforated plate with many holes leads to the formation of small bubbles that hardly break. On the contrary, a perforated sparger with a few big holes induces the production of big bubbles in the lowest part of the column, enhancing breakage. In this second case, due to breakage, bubble size de-
\end{abstract}

\footnotetext{
${ }^{*}$ Corresponding author

Email address: luca.gemello@polito.it (Luca Gemello)
} 
creases quickly along the axial position until an equilibrium bubble size is reached. The presence of additives is used to further investigate coalescence and breakage phenomena, given that it generally suppresses coalescence and highlights the kinetics of breakage. In this work, a suitable set of breakage and coalescence kernels is proposed, capable of providing predictions in good agreement with experimental data for different bubble columns geometries, gas flow rates and additive concentrations.

Keywords: Bubble columns, Heterogeneous regime, QMOM, Population balance modelling

\section{Introduction}

Bubble column reactors play an important role in chemical engineering applications. These reactors are frequently used in the case of chemical reactions with slow kinetics such as alkylation, Fischer-Tropsch synthesis, fermentation, hydrogenation, hydroformylation, oxidation, chlorination, desulfurization and coal liquefaction (Chaumat et al., 2007; Ranade, 2002). Bubble column reactors are also used for wastewater treatment, for cell cultures and for the production of organic acids and yeasts (Tisnadjaja et al., 1996). Industrially these systems often operate in the churn-turbulent flow regime, also called "heterogeneous regime". Local flows, turbulence, gas holdup and bubble size are linked in a complex way with the operating conditions and the design variables.

Computational fluid dynamic (CFD) simulations are a fundamental tool for the study of these systems (Jakobsen et al., 2005). Their behaviour can be determined over a wide range of validity. The interactions between the 
gaseous dispersed phase and the liquid continuous phase dominate gas-liquid systems. The dispersed phase is formed by bubbles that interact due to their chaotic movement under the heterogeneous regime. The bubble size depends on operating conditions, global hydrodynamics and turbulence.

Eulerian-Eulerian RANS methods, also called "Two-Fluid Model", can be used to simulate heterogeneous flows with a relatively low computational cost. This approach is often used for engineering applications. The quality of the results is based on the validity of sub-grid physical models that are implemented to represent turbulence and interfacial forces. One important limitation of Eulerian-Eulerian classical CFD modelling is due to the assumption that every bubble has the same size and that this is an input parameter of the simulation. The bubble size estimation is not however easy: many correlations are available in the literature, strongly depending on the studied system, each one giving different results. Moreover, the use of a single bubble size hinders the prediction of local hydrodynamic properties. Bubble polydispersity causes considerable variations in the interfacial area. These variations influence the rate of exchange of mass, momentum and energy between the two phases (i.e. continuous and dispersed) and between the multiphase flow and the external boundaries. A population balance model (PBM) overcomes this limitation. The use of PBM makes the CFD model more predictive. The transport of separated classes of bubbles, although displaying some advantages, is time-consuming. To overcome this drawback, the method of moments suggests studying the moments of the distribution. To get closure equations, one possibility is to use a Quadrature-Based Moment Methods (QBMM), that results from the original method called 
Quadrature Method of Moments (QMOM) (Marchisio and Fox, 2013). The bubble size distribution (BSD) depends on the coalescence and breakage phenomena, that also depends on operating conditions and turbulence. These phenomena are mainly studied for low gas velocities (Falzone et al., 2018). There is a lack of models suitable for the heterogeneous regime.

Indeed, until recently, it was not possible to measure properly bubble sizes beyond low gas hold-up, as suggested by McClure et al. (2017). The few measurements in the heterogeneous regime are principally obtained with multi-point needle probes, as proposed by Xue (2004), Chaumat et al. (2007), McClure et al. (2015) and McClure et al. (2016). This technique is suitable for bubbles having almost vertical trajectories. In the case of the heterogeneous regime, the accuracy is low, since the bubbles have chaotic trajectories (McClure et al., 2013). Despite this limitation, multi-point probes have the advantage of accessing both the bubble chord distribution and the bubble velocity. The cross-correlation (CC) technique proposed by Raimundo (2015) can overcome the limitations of chord-based measurement techniques. This method does not depend on bubble trajectories and it is suitable for the heterogeneous regime (Raimundo, 2015; Raimundo et al., 2016). This technique is complementary to the existing ones as it does not provide chord distributions, but the Sauter mean diameter for every column position. It gives a correct mean bubble size for any spatial position, whereas multi-probe techniques deliver acceptable bubble chord distributions only close to the centre of the columns, where the bubbles rise vertically.

In order to study and validate coalescence and breakage phenomena, the experimental data obtained by Gemello et al. (2018a) are adopted. These 
experiments were carried out in a wide range of superficial gas velocities, from $0.03 \mathrm{~m} / \mathrm{s}$ to $0.35 \mathrm{~m} / \mathrm{s}$. Global and local gas volume fractions, gas and liquid velocities and bubble size were investigated with demineralised water and by adding different ethanol concentrations, employed to reduce bubble coalescence. Furthermore, Gemello et al. (2018a) investigated two different spargers, generating very different initial bubble sizes. Additives and spargers effects are able to dissociate breakage and coalescence models, as they offer the possibility to generate flows that are locally dominated by breakage or coalescence.

Breakage and coalescence phenomena depend strongly on the operating conditions. Different mechanisms are involved in bubble breakage as shown by Liao and Lucas (2009) and the main mechanism is linked to the turbulent fluctuations at the scale of the bubbles. On the other hand, the coalescence kernel is divided between collision frequency and coalescence efficiency, as stated by Liao and Lucas (2010). Collisions are caused by several phenomena and the main one is due to the turbulent fluctuations. The coalescence efficiency can be modelled with three methods: the film drainage model, the energy model and the critical approach velocity model; the first model is the most common for the homogeneous regime. Existing breakage and coalescence models were developed and validated for the homogeneous regime, while there is a lack of kernels suitable for the heterogeneous one. The main goal of this work is to find breakage and coalescence kernels that can be used to simulate the bubble size for both the homogeneous and the heterogeneous regimes. In this work, the breakage and coalescence phenomena are validated under the heterogeneous flow regime, providing a 
suitable and innovative set of models that can predict correctly the bubble size over a wide range of operating conditions. For reaching this goal, 3D transient Eulerian-Eulerian CFD simulations with ANSYS Fluent are carried out. Starting from the experimental data of Gemello et al. (2018a) obtained with the CC technique (Raimundo, 2015), the existing breakage and coalescence kernels, suitable for the homogeneous regime, are tested for the heterogeneous regime. Under the heterogeneous regime, most of the existing models are not valid and it is necessary to combine coalescence and breakage kernels that can be extended for both the homogeneous and the heterogeneous regimes.

The main objective of this work is to provide a set of models able to predict the bubble size in bubble columns under the heterogeneous flow regime. Unsteady three-dimensional Eulerian-Eulerian CFD simulations are coupled with a PBM. Bubble breakage and coalescence phenomena are studied. Experimental data were collected in order to validate the model; these data were presented in Gemello et al. (2018a). The quadrature method of moments is adopted as PBM in order to predict bubble size. A set of breakage and coalescence kernels is proposed to achieve this goal of the work. It ought to be able to predict the bubble size for different operating conditions, with a focus on the heterogeneous flow regime.

\section{Population Balance Modelling}

The dispersed phase consists of bubbles identified by a number of coordinates. The Number-density functions (NDF) contains the information about the population of bubbles (Marchisio and Fox, 2013) and the PBM 
describes its evolution through the solution of the so-called population balance equations (PBEs). In this work, a univariate PBE has been considered, with bubble size and internal coordinates, that is solved with QMOM. The final governing equation is written as follows:

$$
\frac{\partial}{\partial t}\left(\rho_{g} m_{k}\right)+\nabla \cdot\left(\rho_{g} m_{k} \mathbf{u}_{g}\right)=\rho_{g} S_{m_{k}}
$$

where $\rho_{g}$ is the gas phase density, $\mathbf{u}_{\mathrm{g}}$ is the gas phase average velocity, $m_{k}$ is the moment of order $k$ of the BSD, $S_{m_{k}}$ is the source term for the $k^{\text {th }}$ moment due to coalescence and breakage.

The quadrature approximation is used to calculate the moments of the BSD:

$$
m_{k}=\int_{o}^{\infty} n(L) L^{k} \mathrm{~d} L \approx \sum_{\mathrm{i}=1}^{\mathrm{N}=3} w_{i} L_{i}^{k},
$$

as well as the source term due to coalescence and breakage:

$$
\begin{gathered}
S_{m_{k}}=\frac{1}{2} \sum_{i=1}^{N=3} w_{i} \sum_{j=1}^{N=3} w_{j} h_{i j}\left[\left(L_{i}^{3}+L_{j}^{3}\right)^{k / 3}-L_{i}^{k}-L_{j}^{k}\right] \\
+\sum_{i=1}^{N=3} w_{i} g_{i}\left(\bar{b}_{i}^{k}-L_{i}^{k}\right)
\end{gathered}
$$

where $L_{i}$ are the nodes of the quadrature approximation, $w_{i}$ are the weights of the quadrature approximation, $h_{i j}=h\left(L_{i}, L_{j}\right)$ is the coalescence kernel, $g_{i}=g\left(L_{i}\right)$ is the breakage kernel and $\bar{b}_{i}^{k}$ is the $k^{\text {th }}$ moment of the daughter distribution function $\beta\left(L, L_{i}\right)$ of the bubble $L$ generated by the parent 
bubble $L_{i}$ :

$$
\bar{b}_{i}^{k}=\int_{L_{\min }=0}^{L_{\max }=L_{i}} \beta\left(L, L_{i}\right) L^{k} \mathrm{~d} L .
$$

\subsection{Breakage kernel and daughter distribution function}

Breakage mechanisms can be divided into four groups (Liao and Lucas, 2009; Falzone et al., 2018): turbulent fluctuations, macroscopic shear stresses, shearing-off processes and interfacial slip instability. In turbulent gas-liquid systems, the first group of mechanisms is the most important as, in industrial equipment, the bubble breakage is mainly caused by the turbulent pressure fluctuations along the surface and by the collisions between bubbles and eddies. The most common existing models of breakage due to the turbulent fluctuations consider that the turbulence kinetic energy (Coulaloglou and Tavlarides, 1977; Lee et al., 1987; Luo and Svendsen, 1996; Prince and Blanch, 1990; Martínez-Bazán et al., 1999) or the velocity fluctuations (Alopaeus et al., 2002) are higher than a critical value. Alternatively, a balance between inertial and interfacial forces is considered (Lehr and Mewes, 1999; Lehr et al., 2002) or the different criteria are combined (Wang et al., 2003), as well described by Liao and Lucas (2009).

Renardy et al. (2002) studied the breakage due to the viscous shear forces. These forces cause a gradient of velocity around the bubble interface and deform it, causing bubble breakage if the interfacial tension forces are significantly lower than the liquid viscous stresses (Liao and Lucas, 2009). The shearing-off processes appear in the presence of small bubbles and a large one (also referred as erosive breakage), as stated by Fu and Ishii (2003b,a); they are negligible in air-water systems due to low water viscosity. The breakage would be due to interfacial slip instabilities in absence of a net flow 
of the liquid phase: Rayleigh-Taylor instability happens if a light liquid is accelerated into a heavy fluid, while Kelvin-Helmholtz instability occurs if the density ratio is similar to one and a relative velocity exists. Turbulent fluctuations are assumed to be the dominant breakage mechanism in the case of turbulent flows. For this reason, the other mechanisms are neglected in this work. More details on these mechanisms were presented by Liao and Lucas (2009).

Andersson et al. (2012) stated that bubble breakage is mainly binary if the difference of phase density is high and the viscosity of the dispersed phase is low, as in bubble columns. The daughter distribution function $\beta$ describes the size distribution of the bubbles generated after breakage. This information is necessary to fully describe the breakage phenomenon. The daughter distribution functions can be divided into three groups of models: empirical, statistical and phenomenological. Many daughter distribution functions that can be found in the literature are related to a specific breakage kernel, as detailed by Liao and Lucas (2009).

\subsubsection{Existing models for breakage kernel and daughter distribution function}

One of the first models for turbulent breakage of fluid particles was proposed by Coulaloglou and Tavlarides (1977), resulting in the following expression:

$$
g(L)=c_{1} L^{-2 / 3} \varepsilon^{1 / 3} \exp \left[-\frac{c_{2} \sigma}{\rho_{g} \varepsilon^{2 / 3} L^{5 / 3}}\right] .
$$

where $c_{1}$ and $c_{2}$ are numerical constants.

The turbulence dissipation rate employed in the kernel is damped by the 
presence of the dispersed phase:

$$
g(L)=c_{1} L^{-2 / 3} \frac{\varepsilon^{1 / 3}}{1+\alpha_{g}} \exp \left[-\frac{c_{2} \sigma\left(1+\alpha_{g}\right)^{2}}{\rho_{g} \varepsilon^{2 / 3} L^{5 / 3}}\right]
$$

Alopaeus et al. (2002) corrected the model of Narasimhan et al. (1979), considering a dependency on turbulence dissipation rate, obtaining an expression for droplets in liquid. Laakkonen et al. (2006) modified the Alopaeus et al. (2002) kernel in order to use it also for gas-liquid systems, substituting the gas viscosity with the liquid one and obtaining the following expression:

$$
g(L)=c_{3} \varepsilon^{1 / 3} \operatorname{erfc}\left(\sqrt{c_{4} \frac{\sigma}{\rho_{l} \varepsilon^{2 / 3} L^{5 / 3}}+c_{5} \frac{\mu_{l}}{\sqrt{\rho_{l} \rho_{g}} \varepsilon^{1 / 3} L^{4 / 3}}}\right)
$$

where $c_{3}, c_{4}$ and $c_{5}$ are numerical constants. The last two constants do not depend on the studied system and they are obtained from turbulence theory: they are $c_{4}=0.04$ and $c_{5}=0.01$. The first constant was obtained fitting experimental data including air-water systems: it can be assumed equal to $4.0 \mathrm{~m}^{2 / 3}$, as detailed by Petitti et al. (2010).

Luo and Svendsen (1996) proposed a breakage kernel based on the isotropic turbulence theory and probability. Lehr et al. (2002) modelled a breakage kernel, following the approach of Luo and Svendsen (1996), that does not include experimental constants. They provided an analytical solution for the breakage kernel:

$$
g(L)=0.5 \frac{L^{5 / 3} \varepsilon^{19 / 15} \rho_{l}^{7 / 5}}{\sigma^{7 / 5}} \exp \left(-\frac{\sqrt{2} \sigma^{9 / 5}}{L^{3} \varepsilon^{6 / 5} \rho_{l}^{9 / 5}}\right)
$$


Several models have also been proposed to describe the turbulent breakage of the bubbles under the homogeneous flow regime, as shown by Falzone et al. (2018) and Liao and Lucas (2009). They are not presented here since they are suitable only for low gas volume fractions.

Laakkonen et al. (2007) used a very simple daughter distribution function based on a $\beta$-distribution:

$$
\beta\left(L, L_{i}\right)=180\left(\frac{L^{2}}{L_{i}^{3}}\right)\left(\frac{L^{3}}{L_{i}^{3}}\right)^{2}\left(1-\frac{L^{3}}{L_{i}^{3}}\right)^{2},
$$

where $L$ is the size of the daughter bubble and $L_{i}$ is the size of the parent bubble.

If the daughter distribution function proposed by Laakkonen et al. (2007) is adopted, Eq. (4) leads to an analytical solution in the case of binary breakage (Petitti et al., 2010), that may be considered in many cases:

$$
\bar{b}_{i}^{k}=\frac{3240 L_{i}^{k}}{(k+9)(k+12)(k+15)} .
$$

\subsection{Coalescence kernel}

Coalescence is more complex to describe than breakage: the phenomenon involves not only interactions of bubbles with the continuous phase but also interactions with surrounding bubbles once they collide due to the external flow and forces. Coalescence could be studied using empirical or firstprinciple models. The empirical models are simpler but they depend on the experimental set-up and on the geometry and the results can hardly be extrapolated. In first-principle models, the coalescence kernel is generally 
written as the product of:

$$
h\left(L_{1}, L_{2}\right)=h_{0}\left(L_{1}, L_{2}\right) \lambda\left(L_{1}, L_{2}\right),
$$

where $h\left(L_{1}, L_{2}\right)$ is the coalescence kernel, $h_{0}\left(L_{1}, L_{2}\right)$ is the collision frequency and $\lambda\left(L_{1}, L_{2}\right)$ is the coalescence efficiency.

\subsubsection{Collision frequency}

The collision frequency considers the number of collisions in a certain time interval. The total collision frequency is generally considered as the sum of the rates due to different mechanisms. The motion due to the turbulent fluctuations is the most important phenomenon under the studied operating conditions. Other mechanisms, as collisions due to buoyancy (Friedlander, 1977), to mean-velocity gradients (Friedlander, 1977), to wakes (KalkachNavarro et al., 1994; Colella et al., 1999; Hibiki et al., 2001; Wang et al., $2005 a, b)$ and to capture in a turbulent-eddy (Chesters, 1991) are negligible under these operating conditions. More details were presented by Liao and Lucas (2010) and omitted for the sake of brevity in this work.

Bubble collisions due to turbulent eddies were calculated by analogy with the gas kinetic theory by Prince and Blanch (1990), considering the random movement of bubbles in a turbulent flow similar to the random gas motion in the kinetic gas theory (Kennard, 1938). The collision frequency due to the turbulence fluctuations can be calculated as the volume swept by the moving bubble in a certain time interval:

$$
h_{0}\left(L_{1}, L_{2}\right)=S_{12} u_{r e l}
$$


where $u_{r e l}$ is the relative approach velocity between two bubbles. $S_{12}$ is the collision-sectional area of the colliding bubbles and can be calculated as:

$$
S_{12}=\frac{\pi}{4}\left(L_{1}+L_{2}\right)^{2},
$$

where $L_{1}$ and $L_{2}$ are the diameters of the colliding bubbles.

In order to obtain the relative velocity, the most common assumption is that the colliding bubbles share the velocity of an equal-sized eddy, as considered by Coulaloglou and Tavlarides (1977), Prince and Blanch (1990) and Luo (1993). As a consequence, wide eddies transport bubbles without any effect on the relative movement, whereas small eddies do not have sufficient energy to modify the bubble movement. Applying classical turbulence theories and considering the inertial sub-range of isotropic turbulence, the final expression for the relative velocity between colliding bubbles is:

$$
u_{r e l}=\sqrt{2} \varepsilon^{1 / 3} \sqrt{L_{1}^{2 / 3}+L_{2}^{2 / 3}} .
$$

Consequently, the final expression for the collision frequency (Prince and Blanch, 1990) is:

$$
h_{0}\left(L_{1}, L_{2}\right)=c_{1}^{\prime}\left(L_{1}+L_{2}\right)^{2} \varepsilon^{1 / 3} \sqrt{L_{1}^{2 / 3}+L_{2}^{2 / 3}},
$$

where the constant $c_{1}^{\prime}$ depends on the studied system and varies in the range of $0.28-1.11$. $c_{1}^{\prime}$ equal to 0.28 is common for bubble columns. This equation is widely used to compute the collision frequency.

Bubbles larger than the turbulence integral length scale $\mathcal{L}_{t}$ cannot be 
affected by turbulence. Collisions lead to coalescence only if $L_{1}<\mathcal{L}_{t}$. A size ratio of bubble distance to eddies could be considered, while in the original model bubbles and eddies were considered equal-sized (Liao and Lucas, 2010). In order to consider this effect, a parameter could be added to the Eq. (15), as suggested by Wang et al. (2005a,b) and Wu et al. (1998). Wu et al. (1998) obtained this correction factor by assuming that average eddies and bubbles have the same size order:

$$
\Pi=\left[1-\exp \left(-c_{\Pi} \frac{\alpha_{\max }^{1 / 3} \alpha_{g}^{1 / 3}}{\alpha_{\max }^{1 / 3}-\alpha_{g}^{1 / 3}}\right)\right]
$$

where the constant $c_{\Pi}$ depends on the studied system and is equal to 3 for air-water systems.

Another important correction that should be considered is the reduction of the free space for the bubble motion caused by the volume occupied by bubbles, leading to an increase in the collision frequency. Many correlations (Wu et al., 1998; Hibiki and Ishii, 2000; Lehr et al., 2002; Wang et al., 2005b) are available in order to consider this effect, adding a new parameter $\gamma$ (Liao and Lucas, 2010). One of the most common correlations was proposed by Wang et al. (2005b):

$$
\gamma=\frac{\alpha_{\max }}{\alpha_{\max }-\alpha_{g}}
$$

with $\alpha_{\max }=0.8$.

Another correlation was suggested by Lehr et al. (2002):

$$
\gamma^{\prime}=\exp \left[-\left(\frac{\alpha_{\max }^{1 / 3}}{\alpha_{g}^{1 / 3}}-1\right)^{2}\right]
$$


with $\alpha_{\max }=0.6$.

The final equation for the collisions efficiency proposed by Wang et al. $(2005 b)$ is:

$$
h_{0}\left(L_{1}, L_{2}\right)=c_{2}^{\prime} \gamma \Pi\left(L_{1}+L_{2}\right)^{2} \varepsilon^{1 / 3} \sqrt{L_{1}^{2 / 3}+L_{2}^{2 / 3}},
$$

with $c_{2}^{\prime}=\sqrt{2} \pi / 4$.

Lehr et al. (2002) proposed the same general expression without $\Pi$ and using $\gamma^{\prime}$ instead of $\gamma$.

\subsubsection{Coalescence efficiency}

Only some collisions lead to coalescence: some colliding bubbles separate after the collision or bounce off each other. Efficiency can be divided into the three main physical models proposed: the film-drainage model, the energy model and the critical velocity model.

The film-drainage model is the most popular for the homogeneous flow regime. It is based on the idea that two bubbles, after colliding, could coalesce together only if the thin film of liquid that is trapped between the bubbles is drained quickly (Shinnar and Church, 1960). Coulaloglou (1975) proposed to describe the efficiency as:

$$
\lambda\left(L_{1}, L_{2}\right)=\exp \left(-\frac{t_{D}}{t_{C}}\right),
$$

where $t_{D}$ is the drainage time and $t_{C}$ is the contact time.

Different regimes of drainage can be identified according to the rigidity of the bubbles and the mobility of the interfaces (immobile, partially mobile or 
fully mobile). Fluid particles can be assumed rigid when the viscosity is very high or when the diameter of the bubbles is smaller than $1 \mathrm{~mm}$ (Jeffreys and Davies, 1971). Bubbles bigger than $1 \mathrm{~mm}$ are considered deformable. The mobility of the interface depends on the chemical composition of the liquid phase. For immobile interfaces, typical of contaminated systems, the velocity profile during film drainage is parabolic. Mobile interfaces are typical of clean air-water systems.

By defining the contact time as inversely proportional to the strain rate, the following expression is obtained (Coulaloglou and Tavlarides, 1977):

$$
\lambda\left(L_{1}, L_{2}\right)=\exp \left[-c_{3}^{\prime} \frac{\mu_{l} \rho_{l} \varepsilon}{\sigma^{2}}\left(\frac{L_{1} L_{2}}{L_{1}+L_{2}}\right)^{4}\right] .
$$

This expression can be corrected for relatively high gas fractions:

$$
\lambda\left(L_{1}, L_{2}\right)=\exp \left[-c_{3}^{\prime} \frac{\mu_{l} \rho_{l} \varepsilon}{\sigma^{2}\left(1+\alpha_{g}\right)^{3}}\left(\frac{L_{1} L_{2}}{L_{1}+L_{2}}\right)^{4}\right] .
$$

Lee et al. (1987), Chesters (1991) and Luo (1993) proposed different expressions for immobile bubbles, partially mobile interfaces and fully mobile interfaces. 
Wang et al. (2005b) proposed instead to use the following equation:

$$
\lambda\left(L_{1}, L_{2}\right)=\exp \left\{\begin{array}{c}
-\frac{\sqrt{0.75\left[1+\left(\frac{L_{1}}{L_{2}}\right)^{2}\right]\left[1+\left(\frac{L_{1}}{L_{2}}\right)^{3}\right]}}{\left[\frac{\rho_{g}}{\rho_{l}}+c_{\mathrm{VM}}\right]\left[1-\frac{L_{1}}{L_{2}}\right]^{3}} \\
\left.\sqrt{\frac{\rho_{l} L_{1}\left(u_{b}, 1^{2}+u_{b}, 2^{2}\right)}{\sigma}}\right\},
\end{array}\right.
$$

where $c_{\mathrm{VM}}$ is the virtual mass coefficient.

The energy model assumes that the coalescence is controlled by turbulent forces. During an energetic collision, the approach velocity of two colliding bubbles exceeds a critical value and causes an immediate coalescence. Instantaneous coalescence without film drainage is the main mechanism if the approach velocity is greater than this critical value. The energy model was proposed by Howarth (1964) and confirmed by the experiments of Park and Blair (1975) and Kuboi et al. (1972). Sovova (1981) developed a coalescence model considering energetic collisions between drops, considering the ratio between kinetic collision energy and interfacial energy. A similar expression was formulated by Simon (2004).

Another popular approach is the critical velocity model that assumes that coalescence is favoured by gentle collisions, as observed by Doubliez (1991) and Lehr et al. (2002). This empirical model is based on the experimental observation that low approach velocities of the bubbles result in a high fraction of bubbles that coalesce (high efficiency). Lehr et al. (2002) proposed 
the following coalescence efficiency:

$$
\lambda\left(L_{1}, L_{2}\right)=\min \left(\frac{u_{\text {crit }}}{u_{\text {rel }}}, 1\right)
$$

where the relative velocity $u_{r e l}$ is reported in Eq. (14) and the critical approach velocity $u_{\text {crit }}$ depends on the studied system and can be determined experimentally. By fitting with experiments, the $u_{\text {crit }}$ was found to be equal to $0.08 \mathrm{~m} / \mathrm{s}$ for distilled water-air systems (Lehr et al., 2002). This critical value does not depend on the size of the bubbles in the studied range $(3-8 \mathrm{~mm})$. The relative velocity of two bubbles due to turbulent fluctuations usually exceeds the critical approach velocity in bubble columns. The presence of additives in the liquid inhibits the coalescence and causes lower critical approach velocities, as reported by (Lehr et al., 2002). Ribeiro and Mewes (2007) observed that the critical velocity depends on the physical properties, confirming that the critical velocity changes with the coalescent behaviour of the system.

\section{Test cases and setup}

\subsection{Experimental test cases}

The test cases were experimentally investigated and published by Gemello et al. (2018a) and Raimundo et al. (2019). Experiments were conducted in a cylindrical bubble column with a diameter equal to $0.4 \mathrm{~m}$ (Fig. 1a) at atmospheric pressure for a range of superficial gas velocities between $0.03 \mathrm{~m} / \mathrm{s}$ (homogeneous flow regime) and $0.35 \mathrm{~m} / \mathrm{s}$ (heterogeneous flow regime). The initial height of liquid into the column (without gas) was equal to 4 diameters 
(Fig. 1a). The net liquid flow rate was zero during the experiments. Different hydrodynamic properties were measured: gas hold-up, local gas fraction, liquid velocity and Sauter mean diameter of the bubbles. The Sauter mean diameter of the bubbles is defined as the ratio between the total volume of the bubbles and the total surface:

$$
d_{\text {Sauter }}=\frac{\sum d_{V}{ }^{3}}{\sum d_{S}{ }^{2}}=\frac{6 V_{t o t}}{S_{\text {tot }}}
$$

where $d_{V}$ is the diameter of an isovolumic spherical bubble and $d_{S}$ is the diameter of a spherical bubble with the same surface.

The gas was injected through a perforated sparger from the bottom of the column. The sparger had 92 holes of $2 \mathrm{~mm}$ of diameter (Fig. 1b), ensuring a homogeneous distribution of the gas. Subsequently, another perforated sparger was used (7 holes of $9 \mathrm{~mm}$ ) (Fig. 1c): it generated big bubbles close to the bottom and these experimental results are used to decouple breakage and coalescence phenomena in this work.

Gemello et al. (2018a) observed that the main effects of the additives are on the coalescence phenomena compared to the breakage phenomena, thanks to several experiments with different qualities of water: demineralized water (slightly contaminated water), tap water (fully contaminated water) and demineralized water with small percentages of ethanol (0.01\% and $0.05 \%$ in volume). Additives may influence the surface tension but the ethanol concentration in these experiments was too low to have significant variations. Generally, the presence of additives modifies the surface mobility of the bubbles, while the presence of salts modify the double layer forces. The effect on the surface mobility is the most important in presence of low quantities of 


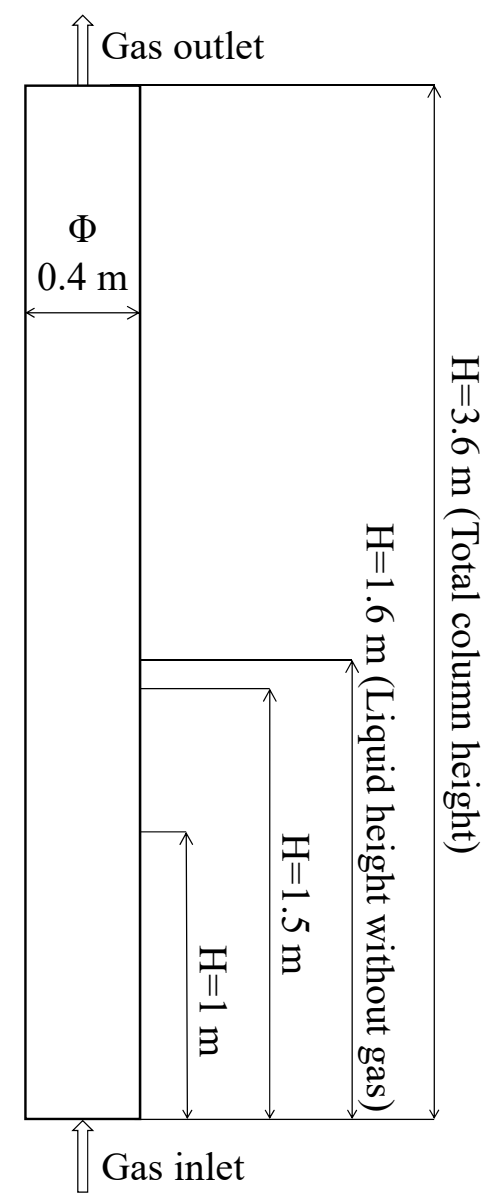

(a) Axial section of the bubble column
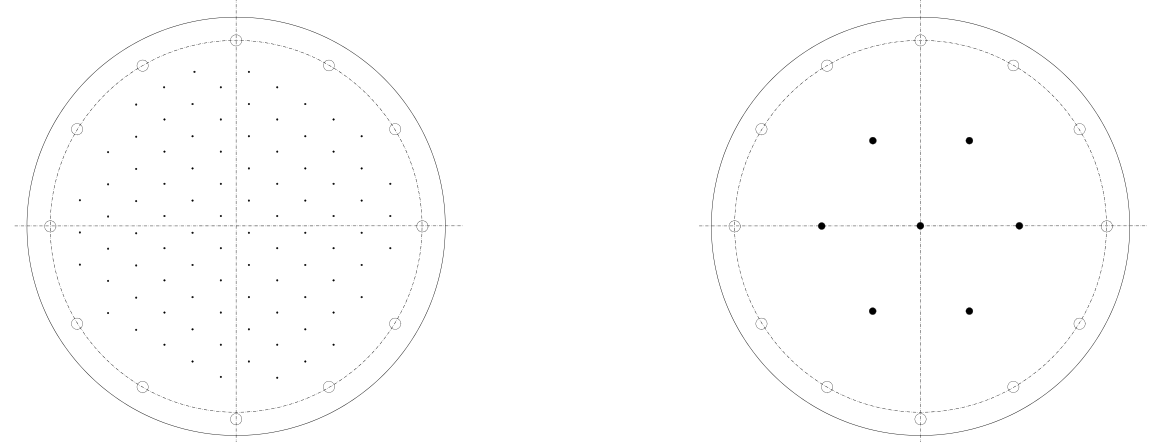

(b) Perforated sparger with 92 holes of $2 \mathrm{~mm}$

(c) Perforated sparger with 7 holes of $9 \mathrm{~mm}$

Figure 1: Bubble column and spargers (Gemello et al., 2018a). 
ethanol. In order to obtain a set of breakage and coalesce kernels that could be used in presence of different additives and contaminants, it is necessary to find a coalescence model that is able to take into account this effect.

The geometrical setups and an overview of the range of operating conditions used for the experiments on the different setups by Gemello et al. (2018a) are reported in Table 1. Every experiment has been repeated on different days to ensure the repeatability of the experimental results.

\begin{tabular}{ll}
\hline Geometry & Cylinder \\
Column diameter & $0.4 \mathrm{~m}$ \\
Column height & $3.6 \mathrm{~m}$ \\
Liquid height without gas & $1.6 \mathrm{~m}$ \\
Superficial gas velocities & $0.03,0.06,0.09,0.16,0.25,0.35 \mathrm{~m} / \mathrm{s}$ \\
Measured properties & Global gas volume fraction \\
& Radial profile of axial liquid velocity $(\mathrm{H} / \mathrm{D}=3.75)$ \\
& Radial profile of local gas volume fraction $(\mathrm{H} / \mathrm{D}=2.5, \mathrm{H} / \mathrm{D}=0.25)$ \\
& Axial profile of local gas volume fraction $($ column center $)$ \\
& Radial profile of Sauter mean diameter $(\mathrm{H} / \mathrm{D}=2.5, \mathrm{H} / \mathrm{D}=0.25)$ \\
& Axial profile of Sauter mean diameter $($ column center $)$ \\
Liquid media & Demineralized water, tap water, Ethanol $0.01 \%$, Ethanol $0.05 \%$ \\
Perforated spargers & 92 holes of $2 \mathrm{~mm}, 7$ holes of $9 \mathrm{~mm}$ \\
\hline
\end{tabular}

Table 1: Geometrical setups and operating conditions used for the different experiments by Gemello et al. (2018a).

\subsection{CFD setup}

CFD simulations are performed using the commercial CFD code ANSYS Fluent 18.0. Under the heterogeneous regime, the simulations must be transient and three-dimensional (3D) as large-scale instabilities are simulated (Ekambara et al., 2005). Instantaneous properties must be averaged over a sample time. This time interval can vary from some seconds up to $100 \mathrm{~s}$ 
depending on the operating conditions and the property of interest. The results of the simulations are averaged over 100 seconds to ensure that every property does not depend on the averaging time. The simulations are carried out by using a Eulerian-Eulerian multiphase method (Jakobsen et al., 2005), solving Reynolds-averaged Navier-Stokes (RANS) equations. Turbulence can be described using several approaches and different models are tested in this work. A Green-Gauss node-based formulation is chosen for the gradient calculation, and the first-order upwind is applied for every convective term, except for volume fraction and momentum, where the QUICK method is used.

The bubble column is modelled as a cylinder with an initial height of the liquid equal to 4 diameters. The total height of the column should be at least twice the initial height of the liquid to ensure that the column can be used for high gas hold-ups (Troshko, 2006). Experimentally gas entered the bubble column through different holes. A real representation of this part of the domain leads to a very fine and irregular mesh, which requires long computational times and it might generate convergence problems. In the present study, a simplified approach is used: the gas enters the domain already mixed with some liquid through a homogeneous equivalent inlet. The perforated plate itself is not simulated: the inlet is set directly on the column-side of the bottom of the cylinder. This choice is justified by the desire to use the developed model for the scale-up of industrial reactors, in which thousands of gas injection holes may be involved. Local gas volume fraction and bubble size were experimentally measured close to the bottom $(H / D=0.25)$. They are imposed as inlet boundary conditions, 
considering also the variations along the radial position. Turbulent intensity and turbulent viscosity ratio are defined as boundary conditions, equal to $5 \%$ and 10 respectively. The gas goes out considering a pressure outlet at the top of the column, with a complete back-flow of gas.

Several numerical meshes are tested. The goal is to find a mesh that provides results that are stable and accurate, avoiding excessive CPU time. A Coopering algorithm in Gambit fulfils these requirements. The mesh obtained with this algorithm is called "cooper mesh" in this work. This mesh is hexahedral, where a rhomboidal $2 \mathrm{D}$ mesh is mapped at the bottom and it is extruded along the entire column. An analysis of the sensibility of the results on the cell size has been done. For the studied test-cases, a mesh with 40,000 cells (Fig. 2) gives satisfactory results with an acceptable computational time, even for larger columns, by scaling-up the mesh. A grid-independence study on this mesh was carried out and presented by Gemello et al. (2018b). Different variables were compared to assess the grid independence.

The interfacial forces dominate these systems (Tabib et al., 2008). The drag force is the most important to be considered. The bubbles have an oblate shape in case of heterogeneous regime and a drag law that can be used also for oblate bubbles, as Tomiyama (1998), is considered in this work. This drag law has the advantage that it takes also into account the water contamination effects. Since high gas volume fractions are considered, it is necessary to use a corrective term, called swarm factor. The swarm factor ought to decrease the drag force effect at high gas fraction. The swarm factor of Simonnet et al. (2008) has this characteristic, but it is not suitable for a 

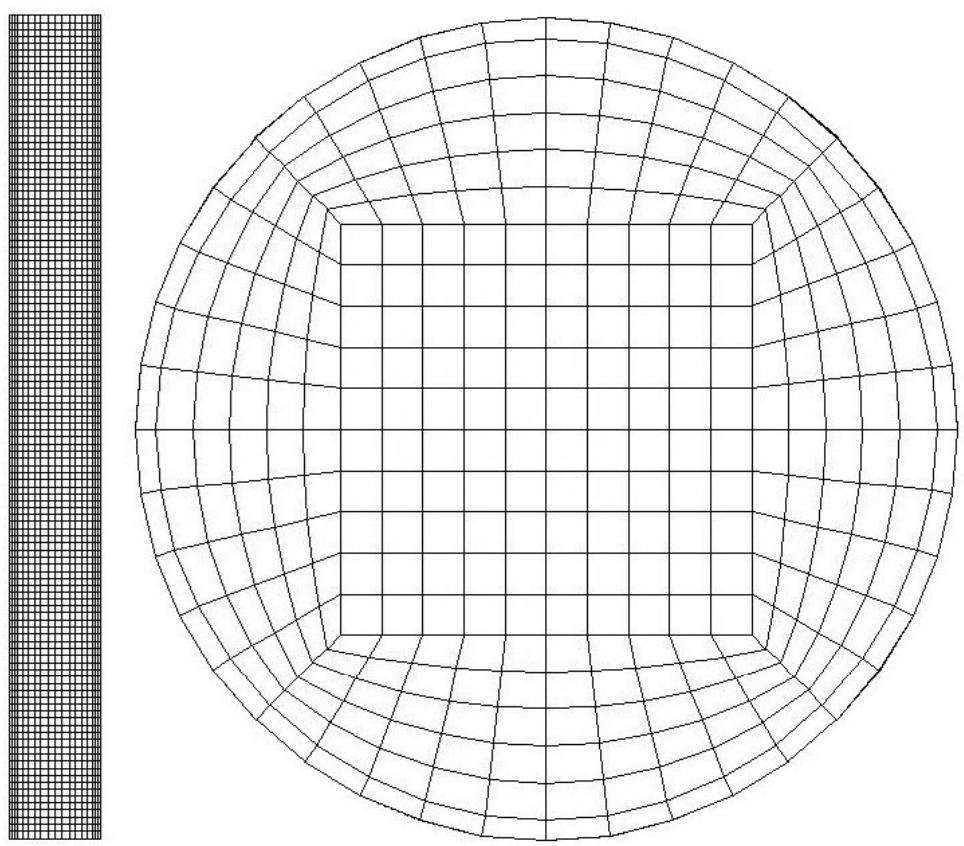

Figure 2: Axial and radial section of the used mesh (Gemello et al., 2018b).

gas volume fraction higher than 30\%. By using the swarm factor of Gemello et al. (2018b), CFD simulations provide a correct hydrodynamic behaviour for a higher gas volume fraction. Considering additional interfacial forces as lift force, virtual mass and wall lubrication force in the heterogeneous regime is still an open question, as stated by Gemello et al. (2018b). Lift force in industrial-scale reactors is far from being understood and its expression in the case of high gas volume fractions is still debated. Lift force is more important when two-dimensional simulations are carried out, as suggested by Joshi (2001). The lift force effect is negligible for three-dimensional twofluid simulations under the heterogeneous regime, as stated by McClure et al. (2013). Gemello et al. (2018b) obtained acceptable results without including the lift force with the operating conditions studied in this work with a two- 
fluid approach. Lift force could have higher effects if a multi-fluid approach was used. Lift force could have a significant effect if the BSD was very large and a multi-fluid approach was adopted, as highlighted by Lucas and Tomiyama (2011). The lift force is neglected in this work since a two-fluid approach is adopted. For similar reasons, virtual mass and wall lubrication force are not considered. Wall lubrication force is important for small pipes, while its effect is minor for large bubble columns.

Different turbulence models were tested by Gemello et al. (2018b) under the same operating conditions and with the same CFD setup with a constant bubble size (without PBM). They stated that the best choice concerning the hydrodynamics is the RNG k- $\varepsilon$ model. For that reason, the RNG k- $\varepsilon$ model is adopted in this work initially. In order to further validate the CFD results, also standard $\mathrm{k}-\varepsilon$ and $\mathrm{k}-\omega$ turbulence models are tested in this work.

In this work, the Quadrature Method of Moments (QMOM) with three nodes is employed, as this is one of the recommended options for univariate PBM. To avoid inconsistent moment sets, the QMOM version suggested by Petitti et al. (2010) is used. For the PBM, six equations are added in Fluent (User Defined Scalars - UDS). The bubbles, that enter the system, have an initial log-normal distribution centred on a mean value and with a fixed variance, according to the work of Petitti et al. (2010). Initially, the three classes of bubbles are considered as a single phase moving with the same velocity, that is calculated by computing the interfacial forces with the Sauter mean diameter (Buffo et al., 2013). To partially overcome this limit, the drag force is computed considering a weighted average of the effects of the size of the three groups of bubbles. The version of QMOM 
that considers the same velocity for each bubble is stable and it calculates the bubbles size for Eulerian-Eulerian simulations with an increase in the computational time of just 30\%. Alternative approaches (Krepper et al., 2008; Lucas and Tomiyama, 2011; Guédon et al., 2017) can consider multiple velocity fields. They have a higher computational cost and they are more difficult to implement due to numerical instabilities. The model proposed in this work is simpler than the others since a single velocity field is required for the dispersed phase (higher robustness). The comparison between these approaches could be an interesting perspective.

In the part of the column above the gas-liquid interface, the liquid fraction is very small and the real primary phase is not the liquid. In the CFD simulations, the liquid phase is considered the primary phase, as it is really under the gas-liquid interface, that is the most important part of the column for the aim of this work. The Dispersed Turbulence Multiphase Model is adopted. This model calculates the turbulence on the primary phase, that is the liquid. The fraction of liquid above the gas-liquid interface is too low and it causes numerical errors. If the gas quantity is over $70 \%$, it becomes the continuous phase and it is then impossible to compute a bubble size. Therefore, the bubble size is calculated only in the zone below the gas-liquid interface. In order to avoid convergence problems at the interface, the KatoLaunder modification is applied in the turbulence kinetic energy equation. The bubble size depends directly on the kernel implemented in the population balance. The main goal of this work is to find a correct kernel for coalescence and breakage in the case of heterogeneous regime. The existing coalescence and breakage kernels, presented above, were studied under the homogeneous 
regime.

\subsection{PBM effects on the global hydrodynamics}

The swarm factor proposed by Gemello et al. (2018b) was validated for a mono-disperse population of bubbles, whereas here it is tested for a polydisperse system. The drag law can be modified in order to consider the effect of the BSD as follows:

$$
\begin{aligned}
F_{D} & \approx \frac{3}{4} \frac{\alpha_{g} \rho_{l}}{\rho_{g}}\left|u_{r e l}\right| u_{r e l} \frac{\int L^{2} C_{D}(L) n d L}{\int L^{3} n d L} \\
& \approx \frac{3}{4} \frac{\alpha_{g} \rho_{l}}{\rho_{g}}\left|u_{r e l}\right| u_{r e l} \frac{\sum_{i=1}^{3} w_{i} L_{i}^{2} C_{D}(L)}{\sum_{i=1}^{3} w_{i} L_{i}^{3}}
\end{aligned}
$$

The water contamination effect can be considered by using the drag law proposed by (Tomiyama, 1998). The water contamination has an effect lower than $10 \%$ on the global gas volume fraction. The hydrodynamics is slightly influenced in case of fixed bubble size. Concerning PBM, water contamination effects can be accounted for by using the critical approach velocity model, as suggested by Lehr and Mewes (1999).

The turbulence properties influence the coalescence and breakage kernels. The main turbulence properties are turbulent viscosity, turbulence kinetic energy and turbulence dissipation rate. The turbulence dissipation rate plays a direct role in the coalescence and breakage models. As detailed above, the simulations are initially carried out by using the RNG $\mathrm{k}-\varepsilon$ model. Other turbulence models ought to be tested, comparing the turbulence properties with experimental data and existing correlations. In this work, different turbulence models are studied: RNG k- $\varepsilon$, standard $\mathrm{k}-\varepsilon$ and $\mathrm{k}-\omega$.

As detailed by Joshi (2001), for a single bubble that rises in liquid, 
the pressure energy is transformed into potential and internal energy. In a turbulent flow regime, the gas energy is converted into turbulence in the liquid phase and finally into internal energy. Yao and Morel (2004) proposed, for low gas volume fractions, that the turbulence due to energy transfer from gas to liquid can be considered by adding source terms in the turbulence properties transport equations (in $\mathrm{k}$ and $\varepsilon$ or $\omega$ equations). The additional turbulence can be considered by adding the contribution of the drag force. In the case of $k-\varepsilon$ models, the source terms for the equation of turbulence kinetic energy and turbulence dissipation rate are respectively equal to:

$$
S_{k}=\frac{C_{D} R e_{b} A_{i} \mu_{l}}{8 L}\left(u_{r e l}\right)^{2}
$$

and

$$
S_{\varepsilon}=\frac{S_{k}}{\left(\frac{L^{2}}{\varepsilon}\right)^{1 / 3}},
$$

where $A_{i}$ is the interfacial area between phases per unit mixture volume. The bubble Reynolds number $\left(R e_{b}\right)$ is defined with respect to the relative velocity between bubble and surrounding liquid $\left(u_{r e l}\right)$.

Troshko and Hassan (2001) used a similar approach, but they stated that only a fraction of the energy due to the drag force is transferred to the liquidphase turbulence, in particular if a higher gas volume fraction occurs. The fraction of gas energy that generates turbulence depends on the operating conditions. The turbulence source terms are multiplied by constants. Similar corrections have been proposed by Simonin and Viollet (1990), Kataoka et al. (1992), Hillmer et al. (1994) and Ranade (1997). The model of Yao and Morel (2004) is the extreme case, where the entire gas-phase energy is converted 
into liquid-phase turbulence. All other models have a lower effect.

Sato and Sekoguchi (1975) proposed a different approach: instead of adding source terms in turbulence equations, the viscosity for the continuous phase is modified:

$$
\nu_{q}=c_{\mu} \frac{k^{2}}{\varepsilon}+0.6 \alpha_{g} L\left|u_{r e l}\right|
$$

where $c_{\mu}$ is the turbulent viscosity constant.

Kataoka et al. (1992), Hillmer et al. (1994) and Ranade (1997) stated that the additional turbulence is compensated by the additional dissipation due to the small-scale interfacial structures. They suggested that the contribution of additional turbulence should almost be ignored. This contribution is far from being well-understood under the heterogeneous flow regime and then it has been initially neglected in this work. The additional turbulence contribution under the heterogeneous regime is not clear, as reported by Joshi (2001), and then it has been neglected in the first part of this work. Afterwards, the other extreme case, i.e. the model of Yao and Morel (2004) (where the entire gas-phase energy is converted into liquid-phase turbulence) is accounted. The absence of this contribution and the Yao and Morel (2004) model represent the extreme cases and all the other models span in between of them. Contributions of the turbulence due to energy phase transfer, following the models proposed by Yao and Morel (2004) and Sato and Sekoguchi (1975), are coupled with the RNG $\mathrm{k}-\varepsilon$ model and tested.

\section{Results and discussion}

Different combinations of coalescence and breakage kernels are tested, as shown in Table 2. Breakage kernel, collision frequency and coalescence 
efficiency are varied, while the daughter distribution function of Laakkonen et al. (2007) is used, because of its analytical solution (Eq. (10)).

\begin{tabular}{|c|c|c|c|}
\hline Case-study & Breakage kernel & Collision frequency & Coalescence efficiency \\
\hline $1(x)$ & $\begin{array}{c}\text { Coulaloglou and Tavlarides (1977) } \\
\text { (Eq. (5)) }\end{array}$ & $\begin{array}{l}\text { Prince and Blanch (1990) } \\
\text { (Eq. (15)) }\end{array}$ & $\begin{array}{c}\text { Coulaloglou and Tavlarides (1977) } \\
\text { (Eq. (21)) }\end{array}$ \\
\hline 2 & $\begin{array}{c}\text { Coulaloglou and Tavlarides (1977) } \\
\text { with damping effect (Eq. (6)) }\end{array}$ & $\begin{array}{l}\text { Prince and Blanch (1990) } \\
\text { (Eq. (15)) }\end{array}$ & $\begin{array}{l}\text { Coulaloglou and Tavlarides (1977) } \\
\text { for high gas fraction (Eq. (22)) }\end{array}$ \\
\hline 3 & $\begin{array}{l}\text { Laakkonen et al. (2006) } \\
\text { (Eq. (7)) }\end{array}$ & $\begin{array}{l}\text { Wang et al. }(2005 b) \\
\quad \text { (Eq. (19)) }\end{array}$ & $\begin{array}{l}\text { Wang et al. }(2005 b) \\
\quad \text { (Eq. }(23))\end{array}$ \\
\hline $4(\bigcirc)$ & $\begin{array}{l}\text { Laakkonen et al. (2006) } \\
\text { (Eq. (7)) }\end{array}$ & $\begin{array}{l}\text { Wang et al. }(2005 b) \\
\quad \text { (Eq. (19)) }\end{array}$ & $\begin{array}{l}\text { Coulaloglou and Tavlarides (1977) } \\
\text { for high gas fraction (Eq. }(22))\end{array}$ \\
\hline $5(\triangle)$ & No breakage & $\begin{array}{l}\text { Wang et al. }(2005 b) \\
\quad \text { (Eq. (19)) }\end{array}$ & $\begin{array}{l}\text { Coulaloglou and Tavlarides (1977) } \\
\text { for high gas fraction (Eq. }(22) \text { ) }\end{array}$ \\
\hline 6 & $\begin{array}{l}\text { Lehr et al. (2002) } \\
\text { (Eq. (8)) }\end{array}$ & $\begin{array}{l}\text { Lehr et al. }(2002) \\
\left.\text { (Eq. (19) with } \gamma^{\prime}\right)\end{array}$ & $\begin{array}{l}\text { Lehr et al. (2002) } \\
\quad \text { (Eq. (24)) }\end{array}$ \\
\hline $7(\diamond)$ & $\begin{array}{l}\text { Laakkonen et al. (2006) } \\
\text { (Eq. (7)) }\end{array}$ & $\begin{array}{l}\text { Wang et al. }(2005 b) \\
\text { (Eq. (19)) with } C_{2}^{\prime}=0.16\end{array}$ & $\begin{array}{l}\text { Lehr et al. (2002) } \\
\quad \text { (Eq. (24)) }\end{array}$ \\
\hline
\end{tabular}

Table 2: Case-studies with different breakage and coalescence kernels.

Initially, simulations are carried out with the typical kernels of the homogeneous regime (case-study 1). This set of kernels will be referred to as "homogeneous" kernels in the remaining part of this work.

The Sauter mean diameter is the first property that should be studied for screening the quality of the kernels: if the Sauter mean diameter is wrong, the kernels are certainly incorrect. The Sauter mean diameter is often the most useful bubble size average for bubble column reactors and it can be directly obtained by the QMOM. The bubble size depends on the coalescence and breakage phenomena and if their models are wrong the bubble size is not correctly predicted. The Sauter mean diameter need to be studied for different operating conditions and in different spatial positions in order to screen the existing coalescence and breakage models. Experimental 
results suggest that the Sauter mean diameter should increase or remain constant when the superficial gas velocity is increased. The homogeneous kernels (case-study 1) provide a wrong behaviour: the Sauter mean diameter decreases while increasing the superficial gas velocity, as shown in Fig. 3. These kernels provide an acceptable prediction of the Sauter mean diameter only for the homogeneous regime; whereas the Sauter mean diameter is underestimated under the heterogeneous regime.

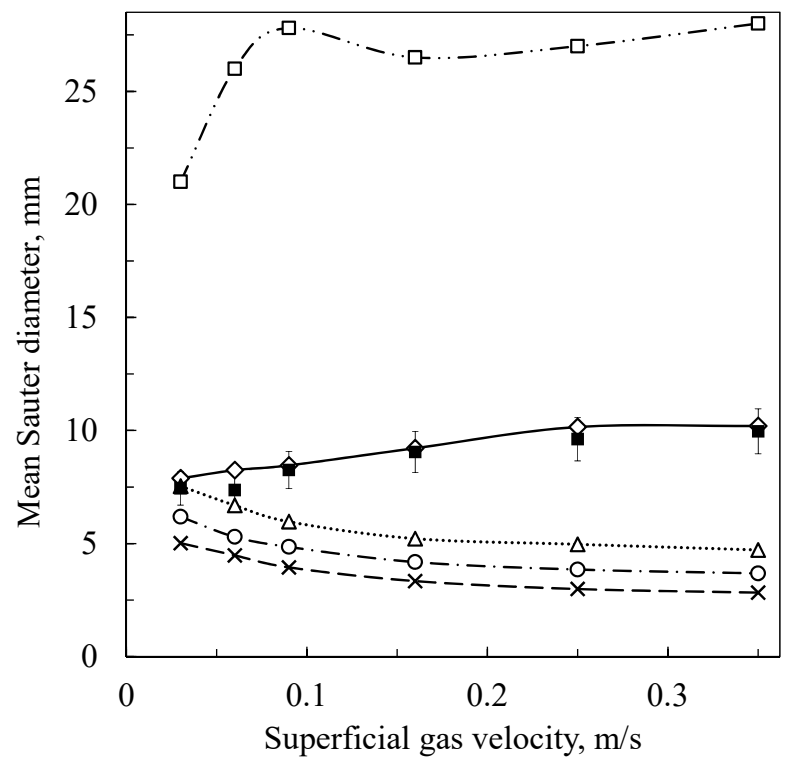

Figure 3: Volume-average Sauter mean diameter at different superficial gas velocities at $\mathrm{H} / \mathrm{D}=2.5$ by using the RNG k- $\varepsilon$ turbulence model. Experimental data (-) versus CFD results obtained with homogeneous kernels (case-study 1 ) (dashed line and $\times$ ), with film drainage velocity model and Laakkonen et al. (2006) breakage (case-study 4) (dash-dot line and $\bigcirc$ ), with film drainage model without breakage (case-study 5) (dotted line and $\triangle$ ), with Lehr et al. (2002) set of models (case-study 6) (dash-dot-dot line and $\square$ ) and with critical approach velocity model and Laakkonen et al. (2006) breakage (case-study 7) (solid line and $\diamond$ ).

Using the homogeneous kernels (case-study 1) a poor prediction of the Sauter mean diameter occurs (Fig. 4): under the heterogeneous regime, the 
experimental profiles are parabolic, whereas the CFD profiles are completely flat. The results shown in Fig. 4 confirm that the case-study 1 cannot be used under the heterogeneous regime.

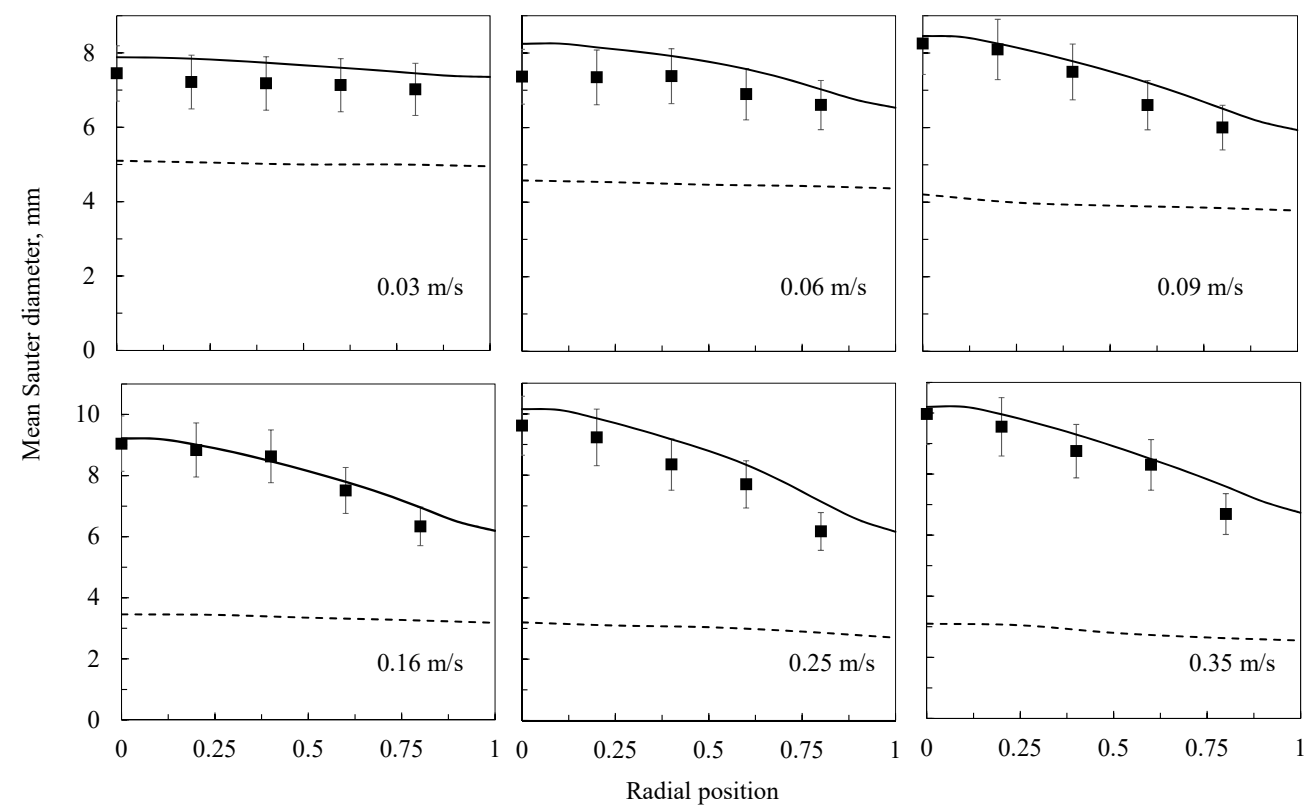

Figure 4: Sauter mean diameter profile at different superficial gas velocities at $\mathrm{H} / \mathrm{D}=2.5$. Experimental data (-) versus CFD results obtained with homogeneous (case-study 1) (dashed line) and global (case-study 7) (solid line) kernels.

Coalescence and breakage can be studied separately in order to understand the source of the discrepancy. It is possible to observe in Fig. 3 that the coalescence kernel predicts a decrease of the Sauter mean diameter if the superficial gas velocity is increased (case-study 5). The efficiency of Coulaloglou and Tavlarides (1977) is not appropriate for the heterogeneous regime. The effect of the turbulent dissipation rate is too high and the coalescence efficiency decreases too much for high $\varepsilon$. The definition of coalescence efficiency based on the film drainage model is not suitable, as the 
Sauter mean diameter decreases if the superficial gas velocity is increased. Corrections for high gas volume fractions are not enough to obtain acceptable results if they are used with a coalescence efficiency based on the film drainage model. The Sauter mean diameter decreases increasing the superficial gas velocity and it is underestimated for case-studies 1, 2, 3 and 4. The Sauter mean diameter values calculated with case-studies 1 and 2 have overlapping lines and these figures would be difficult to read. The same for case-studies 3 and 4. For this reason, case-studies 2 and 3 are not reported in Fig. 3. The critical approach velocity model proposed by Lehr et al. (2002) is then tested. This coalescence kernel provides a correct behaviour for the Sauter mean diameter: it increases with the superficial gas velocity, but the values are incorrect (case-study 6). The Sauter mean diameter is higher than the experimental one. The results obtained with case-study 6 are reported in Fig. 3. This coalescence model seems relevant but the constants ought to be modified.

\subsection{Kernel identification}

The goal is to find a coalescence kernel suitable for gas-liquid systems under several operating conditions, with a focus on the heterogeneous regime. For the heterogeneous regime, the best choice seems to couple the collision frequency of Wang et al. (2005b), the coalescence efficiency of Lehr et al. (2002), the breakage kernel of Laakkonen et al. (2006) and the daughter

distribution function of Laakkonen et al. (2007) (case-study 7). This set of kernels is referred to as "global" kernels in this work.

The critical approach velocity is $0.08 \mathrm{~m} / \mathrm{s}$ in the case of pure water. This value was obtained by Lehr et al. (2002) through experimental observation. 
For tap water (slightly or fully contaminated water) the critical approach velocity is lower, as stated by Lehr et al. (2002). Using the collision frequency of Wang et al. (2005b), the Sauter mean diameter increases with the superficial gas velocity but its values are bigger than experimental data. Numerical constants could be added and fitted with CFD simulations, replacing $\sqrt{2} \pi / 4$ with a constant, as suggested by Buffo (2012). Using this set of kernels, the Sauter mean diameter, averaged in the stable region of the column, slightly increases with the superficial gas velocity and its values are similar to experimental data with a constant equal to 0.16, as shown in Fig. 3.

The CFD radial profiles of the Sauter mean diameter are compared with the experimental data in Fig. 4. The film drainage model provides a wrong Sauter mean diameter profile in the case of the heterogeneous regime: a completely flat profile (case-study 1). The corrected critical approach velocity model provides radial profiles that are very similar to the experimental ones: it provides a parabolic Sauter mean diameter profile in the case of the heterogeneous regime (case-study 7) because of the prediction of opposite gradients of breakage and coalescence efficiencies in the radial direction. The CFD and experimental profiles are in a good agreement for a superficial gas velocity equal to $0.16 \mathrm{~m} / \mathrm{s}$. The profiles are quite correct for every superficial gas velocity. Some discrepancies occur close to the wall. The CFD Sauter mean diameter profile is almost flat for a superficial gas velocity equal to $0.03 \mathrm{~m} / \mathrm{s}$, that can be considered homogeneous flow regime. These results show that it is not necessary to add a complex size-depending lift force to explain the radial gradient of the bubble size. As these preliminary 
results are in accordance with the experiments, they need to be validated by studying the effects of turbulence, initial conditions and scale-up.

\subsection{CFD-PBM simulations validation}

\subsubsection{Hydrodynamics validation}

The hydrodynamic properties were well predicted using a fixed bubble size, as detailed by Gemello et al. (2018b). CFD-PBM coupling can be used to understand if the swarm factor proposed by Gemello et al. (2018b) is suitable for a poly-disperse system. CFD-PBM simulations using the default drag force and the drag force presented in Eq. (26) are compared in the case of the heterogeneous regime. For the operating conditions investigated in this work, this effect is low, as shown in Fig. 5, since the terminal velocity is roughly constant in this size range (Gemello et al., 2018b). This correction on the drag force is robust and the computational time does not change. This effect could be significant in the case of a class of bubble diameter smaller than $2 \mathrm{~mm}$ or bigger than $10 \mathrm{~mm}$. The CFD-PBM global hydrodynamics is correct, as observed by Gemello et al. (2018b) without PBM: gas holdup, local gas fraction (Fig. 5a) and liquid velocity (Fig. 5b) are similar to experimental data. Accordingly, the swarm factor proposed by Gemello et al. (2018b) is considered suitable for a poly-disperse system.

\subsubsection{Effect of turbulence model}

The turbulence dissipation rate could have a strong effect on the coalescence, as detailed above. Several turbulence models have been tested with case-study 7 . The turbulence model choice can influence the turbulence dissipation rate in a significant way, as reported in Fig. 6. The RNG k- 


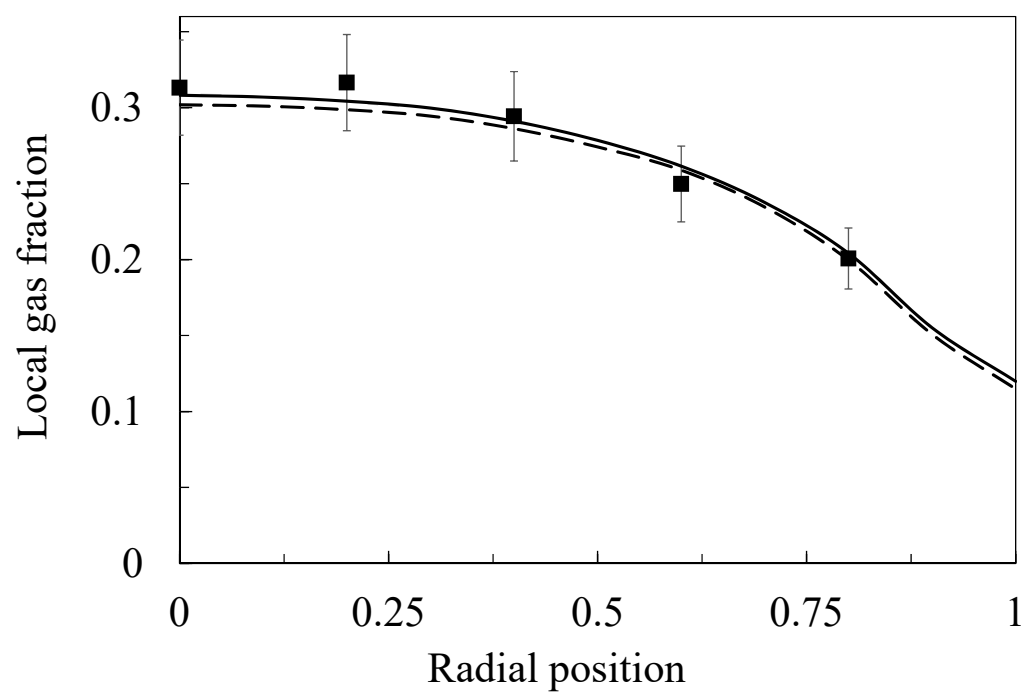

(a) Gas volume fraction at $\mathrm{H} / \mathrm{D}=2.5$.

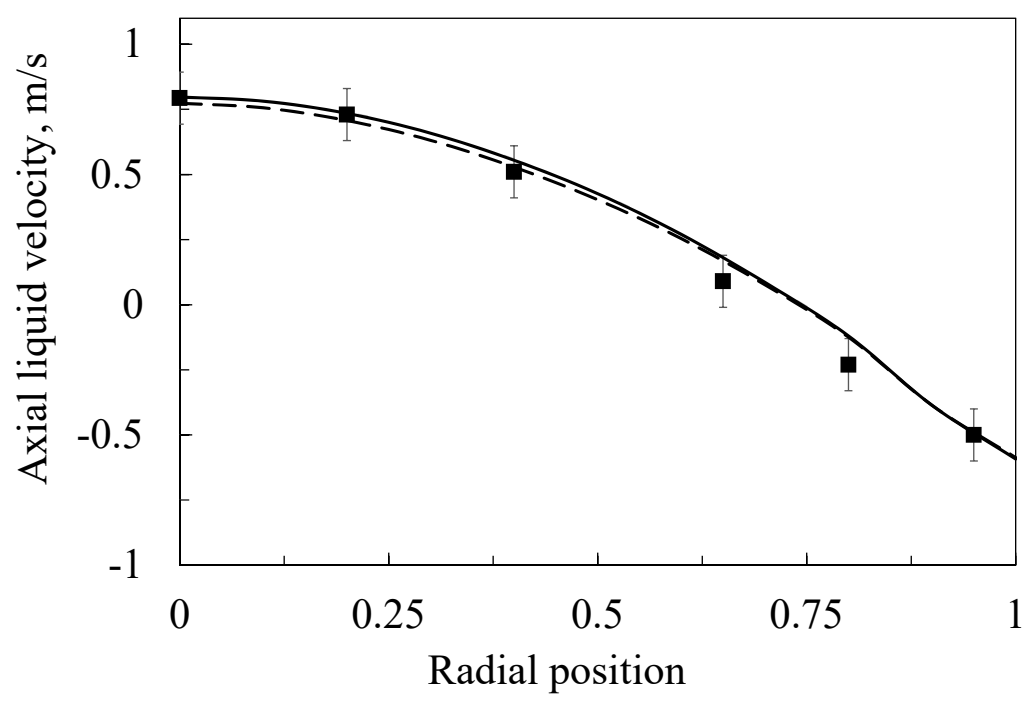

(b) Axial liquid velocity at $\mathrm{H} / \mathrm{D}=3.75$.

Figure 5: PBM effect on the hydrodynamics for a superficial gas velocity equal to $0.16 \mathrm{~m} / \mathrm{s}$. Experimental data obtained by Gemello et al. (2018a) (匹) versus CFD results considering PBM (case-study 7) and the corrected drag force (Eq. (26)) (solid line) and using a fixed bubble size equal to $8 \mathrm{~mm}$ (without PBM) (dashed line) 
$\varepsilon$ model provides a lower turbulence dissipation rate than with the other models. The k- $\omega$ model provides a turbulence dissipation rate that depends more on the radial position. These differences are quite high and the main differences are near the wall. It is important to specify that the wall lubrication force is neglected, for the reasons detailed above.

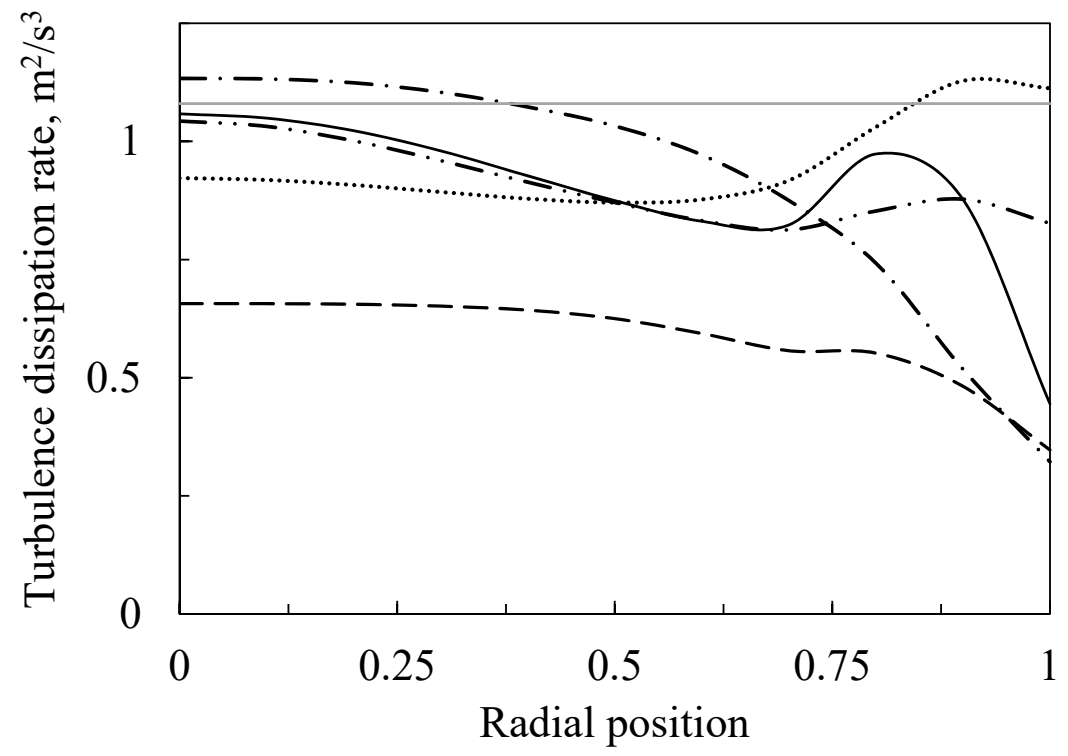

Figure 6: Turbulence dissipation rate with a superficial gas velocity equal to $0.16 \mathrm{~m} / \mathrm{s}$ at $\mathrm{H} / \mathrm{D}=2.5$. Value calculated through Eq. (30) (grey solid line) vs CFD results with different turbulence models: $\mathrm{k}-\omega$ (black solid line), standard k- $\varepsilon$ (dotted line), RNG k- $\varepsilon$ (dashed line) and RNG k- $\varepsilon$ with the corrections of Yao and Morel (2004) (dash-dot line) and Sato and Sekoguchi (1975) (dash-dot-dot line).

Considering an energetic balance, the power consumption per unit of mass in a bubble column is supposed to be equal to the loss of potential energy of the gas per unit of mass, i.e. the turbulence dissipation rate, as considered by Baird (1992) and more recently by Sánchez Pérez et al. (2006). Roels and Heijnen (1980) and Menzel et al. (1990) proposed to calculate the power 
consumption per unit of volume $(P / V)$ with the following formula:

$$
P / V=\frac{1}{\mathrm{H}_{l}} p u_{s g} \ln \left(1+\rho_{l} \frac{\mathrm{g} \mathrm{H}_{l}\left(1-\bar{\alpha}_{g}\right)}{p}\right)
$$

where $\mathrm{H}_{l}$ is the actual height of liquid into the column, $p$ is the external pressure, $u_{s g}$ is the superficial gas velocity, $\rho_{l}$ is the liquid phase density, $g$ is the gravity acceleration and $\bar{\alpha}_{g}$ is the gas hold-up.

For a superficial gas velocity equal to $0.16 \mathrm{~m} / \mathrm{s}$, considering a gas holdup of 0.24 , the turbulence dissipation rate should be equal to $1.08 \mathrm{~m}^{2} / \mathrm{s}^{3}$, slightly higher than the values reported in Fig. 6. The RNG k- $\varepsilon$ model seems to slightly underestimate the turbulence dissipation rate. On the other hand, the $\mathrm{RNG} \mathrm{k}-\varepsilon$ model provides more stable simulations. The contribution of the turbulence due to energy transfer from gas to liquid, following the models proposed by Yao and Morel (2004) and Sato and Sekoguchi (1975) coupled with the RNG $\mathrm{k}-\varepsilon$ model, increases the turbulence dissipation rate, that is more similar to that calculated with Eq. (30), as reported in Fig. 6.

The goal of this work is not to compare the different turbulent properties to suggest the best turbulence model, as more detailed studies and experiments are required on this topic. This part of the work aims to compare the turbulent properties to understand their effects on the coalescence and breakage phenomena and on the bubble size. The effects of the turbulence model on hydrodynamics are not presented because it is not the goal of this part. This analysis was done by Gemello et al. (2018b) on the same CFD setup without PBM. Standard k- $\varepsilon$, Realizable k- $\varepsilon$, RNG k- $\varepsilon$ and k- $\omega$ models were studied. Standard $\mathrm{k}-\varepsilon$ and Realizable $\mathrm{k}-\varepsilon$ models provided numerical errors for higher superficial gas velocities. RNG $k-\varepsilon$ model provided the 
best and the most stable results. More details are reported in the original article. As detailed in Section 4.2.1, the PBM effect on the hydrodynamics is demonstrated to be low with the CFD-PBM approach used in this work. The PBM effect is low for every turbulence model. As consequence, figures on the turbulence model effects on hydrodynamics are omitted. The effect of the turbulence model choice on the Sauter mean diameter is quite low, as shown in Fig. 7. Every turbulence model provides similar results. The RNG k- $\varepsilon$ and $\mathrm{k}-\omega$ models provide similar radial profiles, but the Sauter mean diameter is slightly lower if it calculated with the $\mathrm{k}-\omega$ model. The standard $\mathrm{k}-\varepsilon$ presents some differences next to the wall, with a more pronounced profile. The effect of the turbulence due to energy transfer from gas-phase to liquid-phase on the Sauter mean diameter is low.

This part suggests that the coalescence and breakage kernels studied in this work are usable with different turbulence models. This set of kernels can be used also if the turbulence due to energy transfer from gas to liquid is considered. One possible explanation is that using the critical approach velocity model, the effect of the turbulence dissipation rate on the coalescence kernel is almost null under the operating conditions of the heterogeneous regime. In the collision frequency model adopted in case-study 7, the relative velocity due to the turbulence appears on the numerator. Under the heterogeneous regime, the relative velocity due to the turbulence is higher than the critical approach velocity. In this case, the relative velocity appears on the denominator of the coalescence efficiency (critical approach velocity model). By multiplying collision frequency and coalescence efficiency, the relative velocity, that depends on the turbulence dissipation rate, disappears 


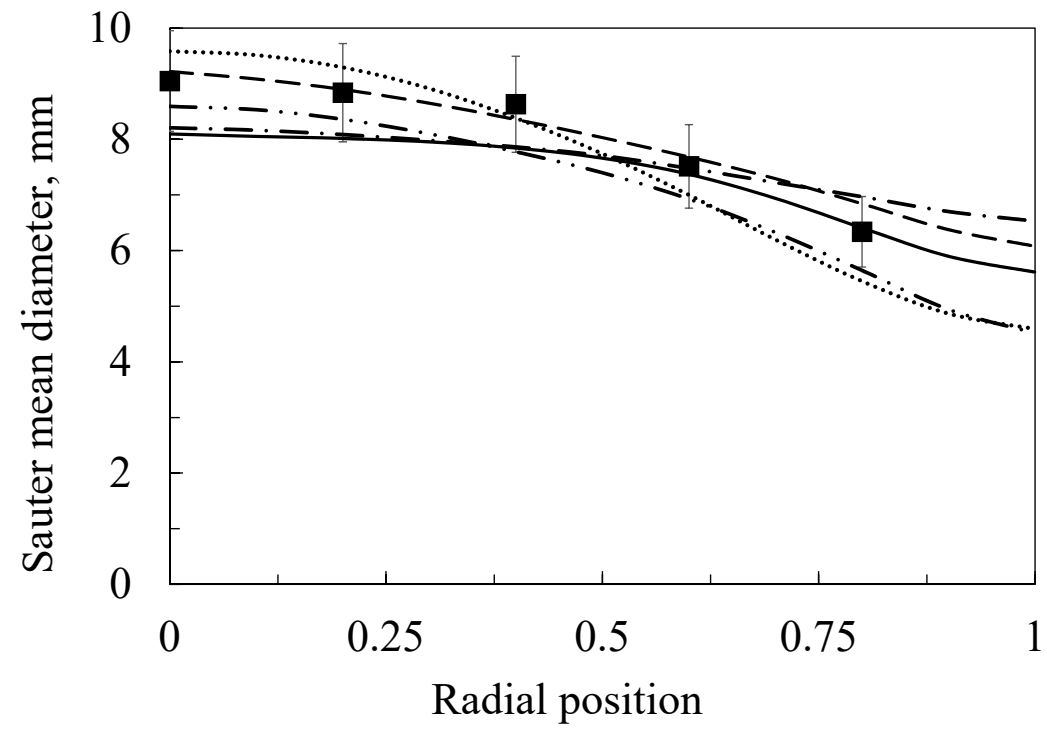

Figure 7: Sauter mean diameter with a superficial gas velocity equal to $0.16 \mathrm{~m} / \mathrm{s}$ at $\mathrm{H} / \mathrm{D}=2.5$. Experimental data obtained by Gemello et al. (2018a) (匹) versus CFD results with different turbulence models: $\mathrm{k}-\omega$ (solid line), standard $\mathrm{k}-\varepsilon$ (dotted line), RNG k- $\varepsilon$ (dashed line) and RNG k- $\varepsilon$ with the corrections of Yao and Morel (2004) (dash-dot line) and Sato and Sekoguchi (1975) (dash-dot-dot line).

in the final formulation if it is higher than the critical approach velocity. This result is a strong point of this set of kernels, as the final models almost do not depend on the relative velocity. The relative velocity was calculated by applying classical turbulence theories and considering the inertial sub-range of isotropic turbulence. In the absence of theories more suited to dense multiphase flows, the classical turbulent theory seems to be the only one that can be used to develop physical kernels. The final combined coalescence model (frequency + efficiency) does not depend on the relative velocity and then the inertial sub-range of isotropic turbulence is overcome. The coalescence efficiency based on the film drainage model tends to dramatically reduce the coalescence when the turbulence dissipation rate 
increases: the exponential function (present in the film drainage efficiency) is stronger than the power function (present in the collision frequency). Since the breakage frequency increases with the superficial gas velocity (which follows the turbulence dissipation rate); whatever the breakage kernel is, the coalescence kernels which predict a significant decrease with the turbulence dissipation rate are clearly not suitable for heterogeneous bubble flows. The critical approach velocity model is the only one that annihilates the turbulence dissipation rate effect, since the relative velocity is simplified. The simulations carried out with the RNG $\mathrm{k}-\varepsilon$ are the most stable and therefore this method has been adopted. The constant for the coalescence frequency of Wang et al. (2005b) has been fitted considering the RNG k- $\varepsilon$ model without the turbulence due to energy transfer from gas to liquid. A similar constant value would be obtained if the best fitting was carried out with other turbulence methods.

\subsubsection{Contamination effects on the bubble size}

Considering the lack of physical descriptors that may explain the different coalescent behaviours, only an empirical modification of the kernel can be attempted. Water contamination effects on the bubble size can be taken into account by lowering the critical approach velocity value. Lehr and Mewes (1999) stated that the surface tension modifies the critical velocity, but the surface tension almost does not change in this work: it means that also other liquid properties influence the critical approach velocity value. In this work, different critical approach velocity values are tested. Gemello et al. (2018a) found that the presence of an additive, e.g. ethanol, lowers the Sauter mean diameter. 
Several values of critical approach velocity are tested and the corresponding volume-average Sauter mean diameter values are calculated with simulations. A linear correlation between critical approach velocity value and volume-average Sauter mean diameter, as observed in Fig. 8, is obtained by using the global kernels (case-study 7) with a superficial gas velocity equal to $0.16 \mathrm{~m} / \mathrm{s}$. If the volume-average Sauter mean diameter is known, it is possible to approximate the critical approach velocity that could be used to obtain a correct bubble size in CFD simulations of bubble columns. The volume-average Sauter mean diameter depends on the concentration and on the nature of the additives. The critical approach velocity model can be used in contaminated systems, as reported in Fig. 9. By using

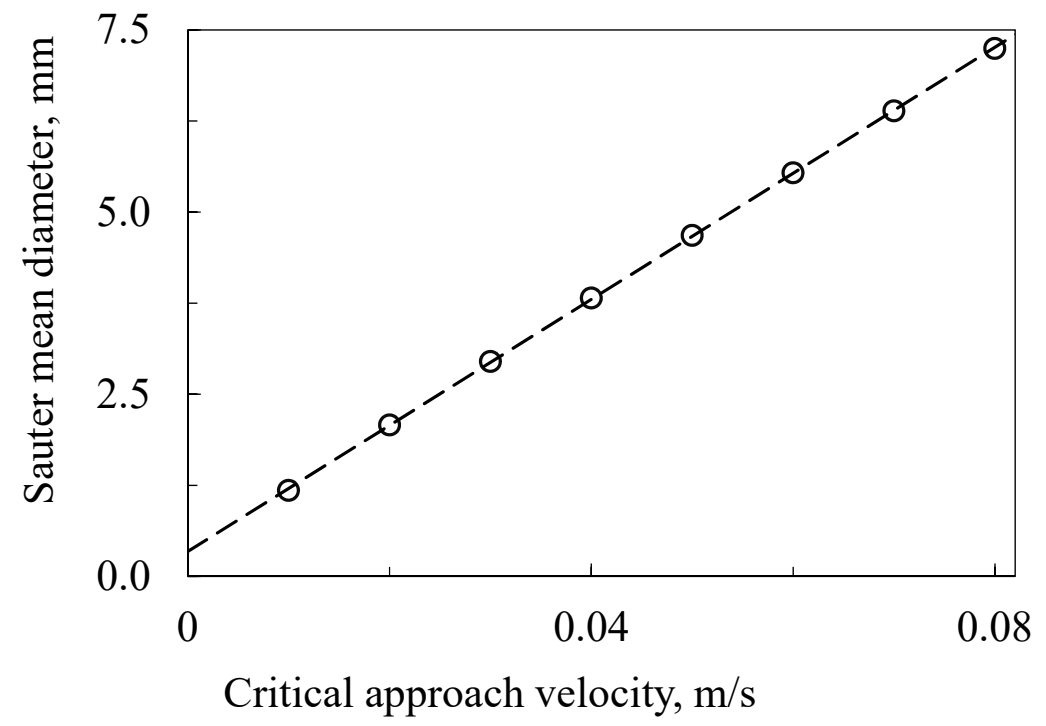

Figure 8: Effect of the critical approach velocity value on the Sauter mean diameter with a superficial gas velocity equal to $0.16 \mathrm{~m} / \mathrm{s}$ (case-study 7 ).

the correct critical approach velocity, it is possible to describe the radial bubble size profile for different ethanol concentrations in demineralised 


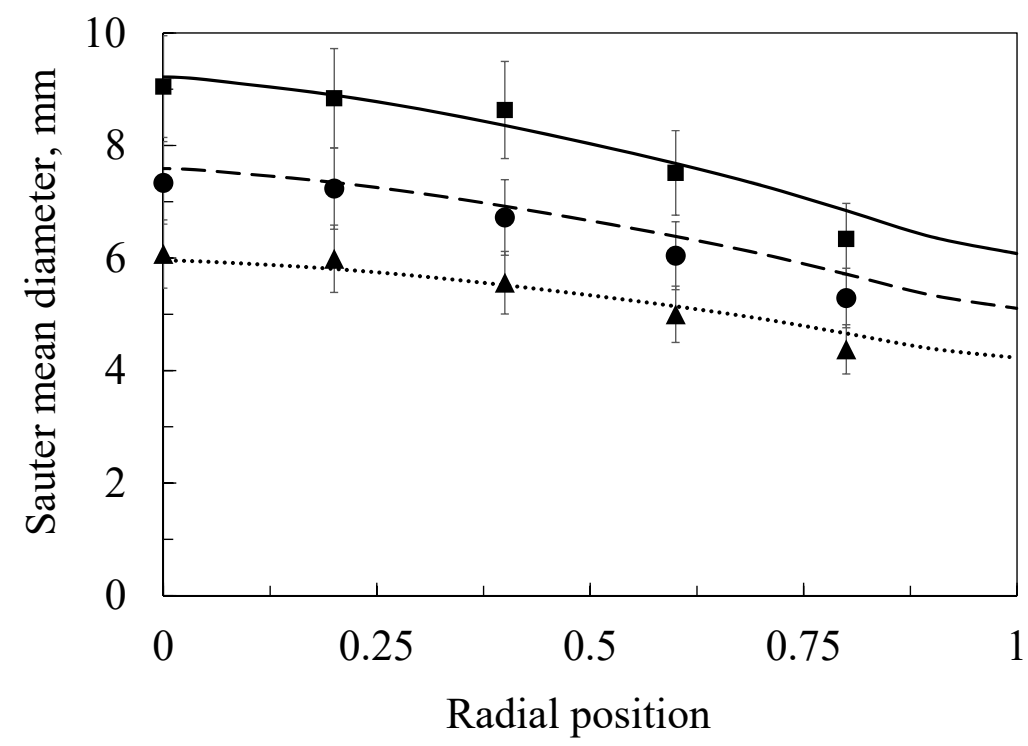

Figure 9: Ethanol effect on the Sauter mean diameter with a superficial gas velocity equal to $0.16 \mathrm{~m} / \mathrm{s}$. Experimental data obtained by Gemello et al. (2018a) with demineralised water $(\mathbf{\bullet})$, ethanol $0.01 \%(\bullet)$ and ethanol $0.05 \%(\mathbf{\Lambda})$ versus CFD results (case-study 7 ) with different critical approach velocities: $0.08 \mathrm{~m} / \mathrm{s}$ (demineralised water) (solid line), $0.065 \mathrm{~m} / \mathrm{s}$ (ethanol $0.01 \%$ ) (dashed line) and $0.05 \mathrm{~m} / \mathrm{s}$ (ethanol 0.05\%) (dotted line).

water. The critical approach velocity value has been obtained starting from the experimental profile and by using the values reported in Fig. 8. Considering the $0.05 \%$ ethanol solution, a critical approach velocity equal to $0.05 \mathrm{~m} / \mathrm{s}$ should be used, while for the $0.01 \%$ ethanol solution and for the contaminated (tap) water, a critical approach velocity equal to $0.065 \mathrm{~m} / \mathrm{s}$ may be considered.

Contaminants affect the surface mobility and then impact the coalescence, while quantitative properties are still missing to describe the effect of ethanol on coalescence. To obtain a fully predictive tool, more experiments with different additives, concentrations and operating conditions are required. Ideally, new experiments and measurements of the liquid properties would 
provide a correlation that links these properties to the volume-average Sauter mean diameter, as partially proposed by Lehr and Mewes (1999). If the proper critical approach velocity is found, the model proposed in this part of the work is able to calculate the radial Sauter mean diameter profile correctly.

\subsubsection{Sparger effects on the bubble size}

Gemello et al. (2018a) carried out experiments by using two different spargers. Initially, a perforated sparger with many small holes (2 mm) was used, that generated bubbles with a Sauter mean diameter in the range 5-10 mm. A sparger with 7 holes of $9 \mathrm{~mm}$ was also used to modify the initial bubble size. These results showed that the sparger influences the bubble size close to the bottom of the column. A perforated sparger with few big holes causes the formation of big bubbles in the lowest part of the column, above the holes. The bubble size decreases quickly along the axial position and the bubbles are almost not influenced by the sparger choice after some centimetres as they reach the equilibrium size quickly. A region of the column (close to the bottom) where the bubble size is higher than the final one is formed, in which the bubble breakage is the predominant phenomenon compared to the coalescence. It is useful for decoupling bubble breakage and coalescence phenomena in order to validate them separately.

The previous simulations have been carried out by approximating the high number of holes of the first sparger (92 holes) with a homogeneous porous plate. In CFD simulations, the gas enters the column already mixed with the liquid with a constant gas volume fraction. The initial bubble size is imposed equal to $7.7 \mathrm{~mm}$ with a narrow log-normal BSD. These approximations are acceptable if the objective is to simulate the sparger with many small holes. 
Similar results are obtained by using an initial homogeneous bubble size slightly smaller $(6.5 \mathrm{~mm})$ or bigger $(10 \mathrm{~mm})$ (Fig. 10). The CFD-PBM model with case-study 7 is able to manage small variations of initial bubble size, as the system reaches an equilibrium between coalescence and breakage.

On the other hand, by approximating the second sparger (7 holes) with a homogeneous porous plate with a high inlet bubble size (e.g. $15 \mathrm{~mm}$ ), the results are incorrect: it is not possible to approximate a sparger with few big holes with a homogeneous porous plate with a high inlet bubble size, as observed in Fig. 10. The bubbles are not able to reach the real equilibrium size obtained in the experiments. The Sauter mean diameter is higher than the real one in the entire column. The CFD-PBM model is not able to manage a homogeneous initial bubble size that is much higher than the real one. It means that the inlet boundary conditions are important in these systems. For studying the sparger effects, new boundary conditions at the inlet ought to be used. The new sparger is created by using bubble size and gas fraction measured with the 7-holes sparger by Gemello et al. (2018a). These properties, measured at $\mathrm{H}=10 \mathrm{~cm}(\mathrm{H} / \mathrm{D}=0.25)$ with the 7-holes sparger, are used to estimate the real value of bubble size and gas fraction. Inlet boundary conditions depend on radial and azimuthal positions with maximum values aligned with the real sparger holes (Fig. 11). In CFD simulations, the effect of this new sparger is important close to the bottom (Fig. 10). Big bubbles disappear quickly by using different initial bubble sizes in correspondence of the real sparger holes. The effect is almost null above $1 \mathrm{~m}$. The behaviour is in accordance with the experimental data. In the experiments, the equilibrium size is reached a little faster, but the results 


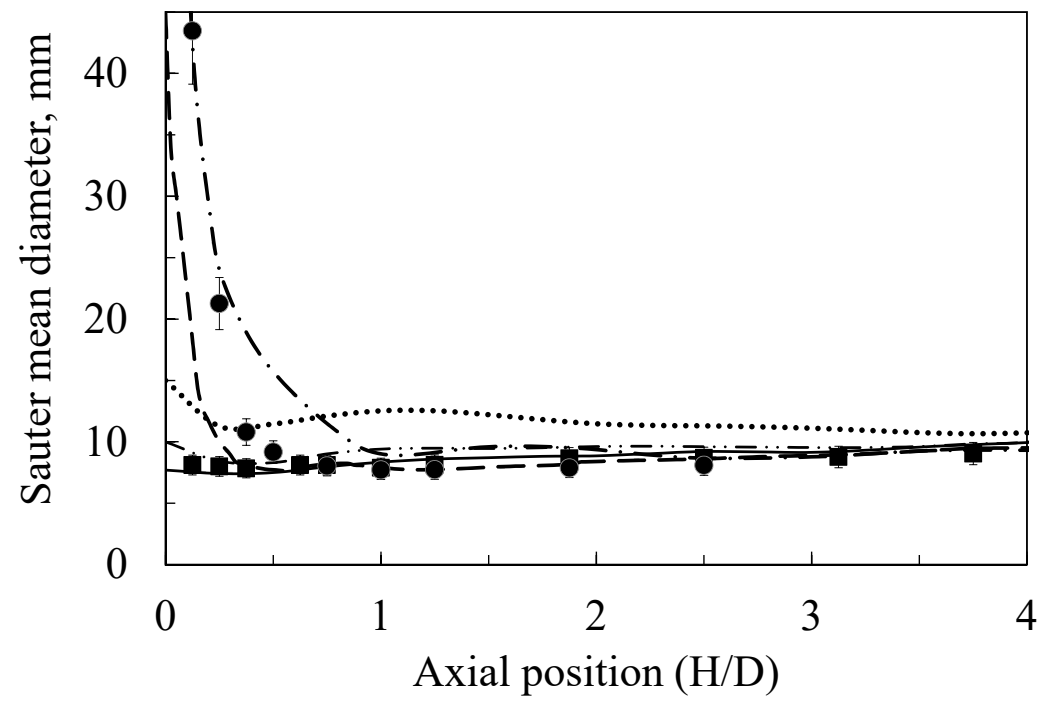

Figure 10: Effect of the sparger on the axial profile at the centre of the column of the Sauter mean diameter with a superficial gas velocity equal to $0.16 \mathrm{~m} / \mathrm{s}$ (case-study 7 ). Experimental data obtained by Gemello et al. (2018a) with the 92-holes sparger (-) and the 7-holes sparger $(\bullet)$ versus CFD results with different spargers: homogeneous sparger with initial Sauter mean diameter equal to $7.7 \mathrm{~mm}$ (solid line), $10 \mathrm{~mm}$ (dash-dot-dot line) and $15 \mathrm{~mm}$ (dotted line) and non-homogeneous sparger with maximum initial Sauter mean diameter equal to $45 \mathrm{~mm}$ (Fig. 11) (dashed line) and $90 \mathrm{~mm}$ (dash-dot line).

are satisfactory. If the CFD simulations use a sparger more similar to the real one, it is possible to study correctly the bubble size in the entire column (Fig. 10). It is important to note that the turbulence dissipation rate is low close to the bottom in the CFD simulations. A detailed study on turbulence models, with a focus on the effects of the inlet turbulence properties, could make it possible to further validate the proposed kernels.

Thanks to these results, it is possible to partially decouple the effect of breakage and coalescence in order to study them separately. In the lowest part of the column, the breakage is the predominant phenomenon and the coalescence is almost negligible. When the breakage is the main 


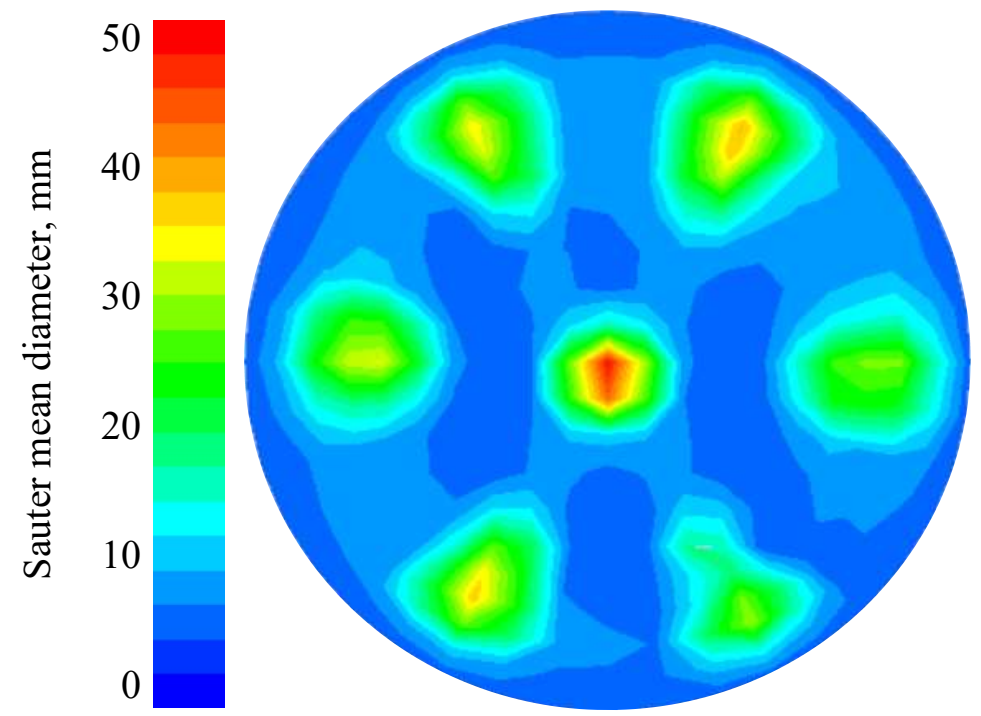

Figure 11: Inlet Sauter mean diameter with the 7-holes sparger.

phenomenon the behaviour is quite correctly predicted, as the bubble size decreases quickly, in accordance with experiments. If the breakage kernel seems satisfactory, the final equilibrium bubble size is correct and the bubble size radial profiles are in agreement with the experiments, it means that also the coalescence kernel can be considered correct. If both the kernels are fine, they can be used both for the new sparger and for the old sparger, where the initial Sauter mean diameter is similar to the final one. The global set of kernels is verified for both spargers, as presented in Fig. 10. Breakage and coalescence kernels can be used in a wide validity range. The inlet boundary conditions are very important in these systems and they influence the hydrodynamics. A homogeneous porous plate can be used to simulate spargers with many small holes. Spargers with few big holes need to be modelled with a non-homogeneous and more realistic boundary condition. 


\subsubsection{Scale-up effect}

Raimundo (2015) carried out experiments by using bubble columns at different scales. Initially, the Sauter mean diameter is measured by using a column with a diameter equal to $0.4 \mathrm{~m}$ and tap water. The measurements are repeated by using columns with a diameter of $1 \mathrm{~m}$ and $3 \mathrm{~m}$. Thanks to these experiments it possible to observe that the Sauter mean diameter is approximately equal or becomes slightly smaller when the column diameter increases (case-study 7). It can be assumed that the behaviour is the same in the case of demineralised water.

In order to study the scale-up effect on the bubble size in the CFD-PBM simulations, the initial column rhomboidal cooper mesh with 40,000 cells is simply scaled up. The column with a diameter equal to $0.4 \mathrm{~m}$ is scaled without other modifications, to keep the number of cells of the mesh constant. Grid-independent tests confirm that this mesh is suitable for the scale-up, as detailed by Gemello et al. (2018b).

By increasing the column diameter, the Sauter mean diameter is approximately constant in the CFD-PBM simulations, as shown in Fig. 12.

It is in accordance with the experimental results obtained by Raimundo (2015) with tap water. Different k- $\varepsilon$-based and k- $\omega$-based methods are tested, leading all of them to similar results. The breakage and coalescence kernels suggested in this work are suitable for the industrial scale-up of bubble columns with different two-equation turbulence models. Different column diameters are then simulated using various initial homogeneous bubble sizes. Keeping in mind that the final objective is the industrial scale-up of bubble columns, in which bubble sizes are not known a priori and often impossible 


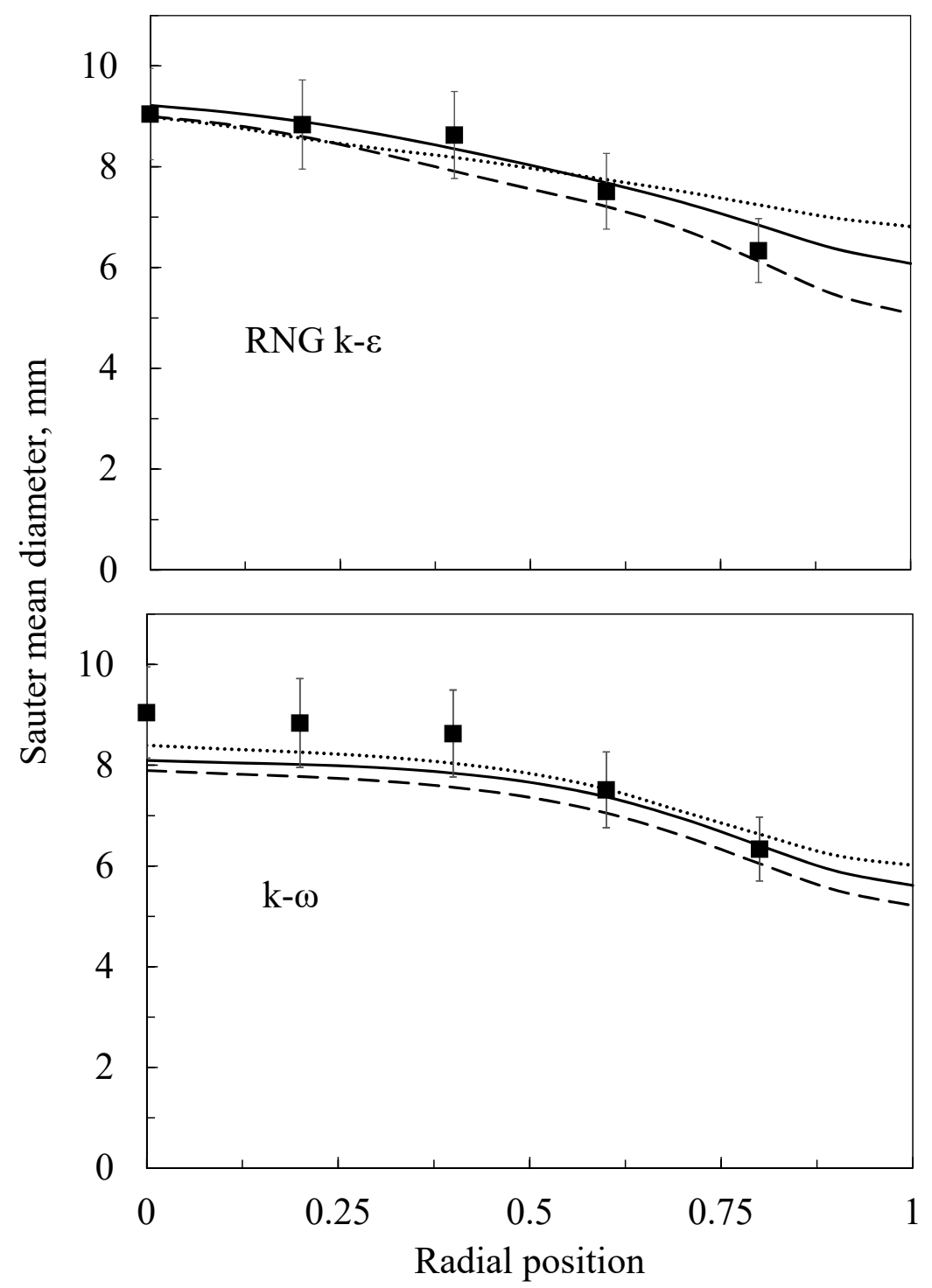

Figure 12: Scale-up effect with a superficial gas velocity equal to $0.16 \mathrm{~m} / \mathrm{s}$ and different turbulence models on the radial profile of the Sauter mean diameter at $\mathrm{H} / \mathrm{D}=2.5$.

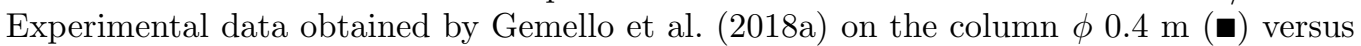
CFD results with different bubble column: $\phi 0.4 \mathrm{~m}$ (solid lines), $\phi 1 \mathrm{~m}$ (dashed lines) and $\phi 3 \mathrm{~m}$ (dotted lines). 
to measure. The inlet bubble size effect is more important in the case of smaller columns. As shown in Fig. 13, the CFD-PBM model can manage small variations of inlet bubble size inside the experimental range. By using an initial bubble size slightly lower than the previous one, equal to $6.5 \mathrm{~mm}$, the bubble size is almost the same above a height of $1 \mathrm{~m}$. By using an initial bubble size equal to $10 \mathrm{~mm}$, the bubble size is slightly higher, but it is still acceptable.

The CFD bubble sizes are compared with the experimental data collected by Gemello et al. (2018a) with demineralised water in the column $\phi 0.4 \mathrm{~m}$ (Fig. 13). Experimental data with demineralised water and industrial-scale columns should be collected to optimize these results. In the biggest columns, the time that a bubble needs to reach $\mathrm{H} / \mathrm{D}=2.5$ is longer than in the smallest columns and the equilibrium bubble size is reached with a wider range of inlet bubble sizes. Consequently, in the case of CFD-PBM simulations of industrial bubble columns, the lack of knowledge of the initial bubble size does not significantly affect the bubble size estimation.

\section{Conclusions and perspectives}

Bubble coalescence and breakage play a fundamental role in the CFD study of hydrodynamics and transfer phenomena for bubble columns under industrial operating conditions. Computational fluid dynamic (CFD) simulations are frequently used for the study of these systems. Population balance modelling (PBM) is coupled with CFD simulations to account for coalescence and breakage phenomena.

Several mechanisms are involved in coalescence and breakage phenomena. 

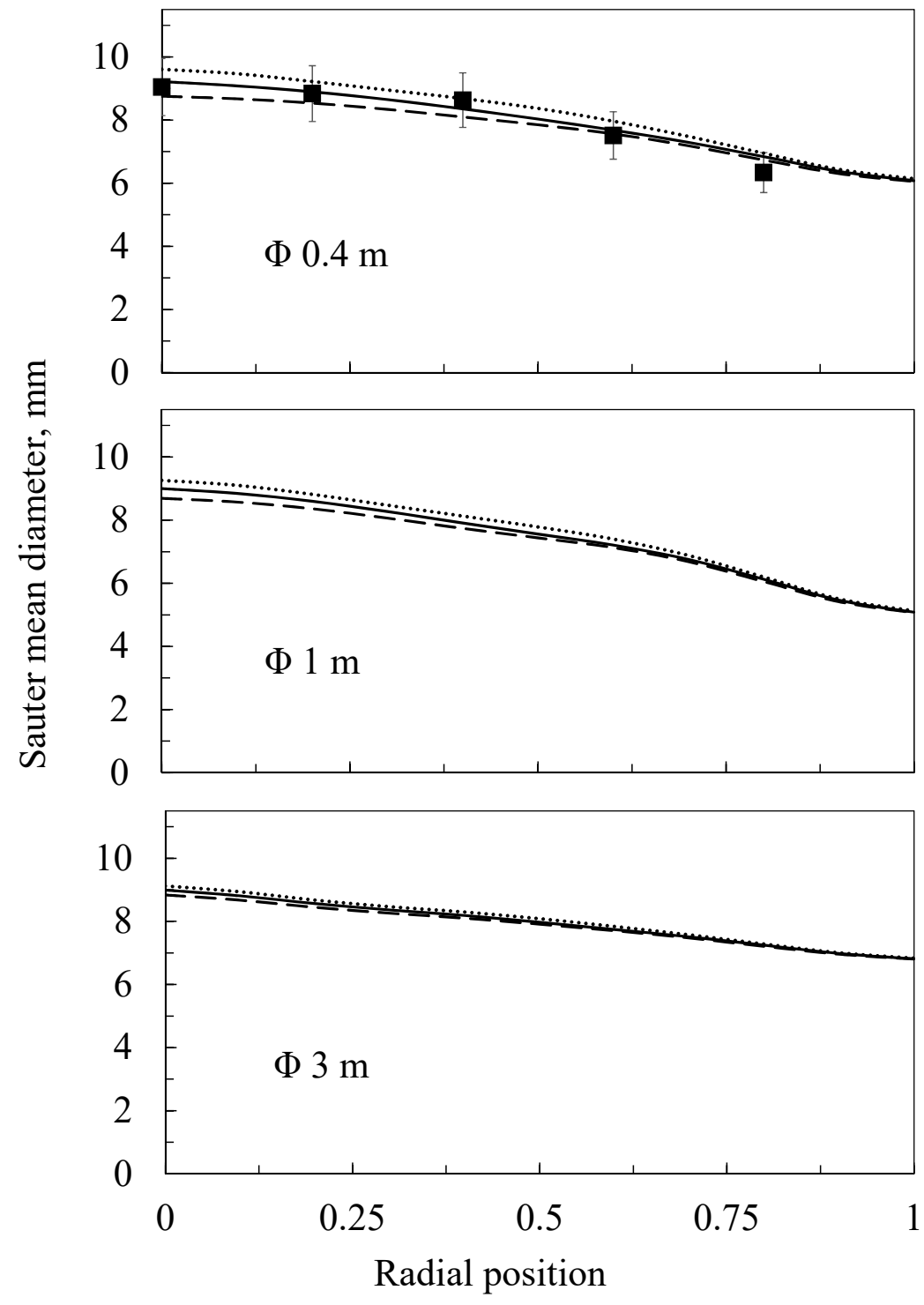

Figure 13: Effect of inlet bubble size (homogeneous sparger) for different bubble columns with a superficial gas velocity equal to $0.16 \mathrm{~m} / \mathrm{s}$ on the radial profile of the Sauter mean diameter at $\mathrm{H} / \mathrm{D}=2.5$. Experimental data obtained by Gemello et al. (2018a) on the column $\phi 0.4 \mathrm{~m}$ (ם) versus CFD results with different inlet bubble sizes: $6.5 \mathrm{~mm}$ (dashed lines), $7.7 \mathrm{~mm}$ (solid lines) and $10 \mathrm{~mm}$ (dotted lines). 
The main mechanisms are linked to turbulent fluctuations. The coalescence kernel is divided into collision frequency and coalescence efficiency. The coalescence efficiency can be studied through three methods: the film drainage model, the energy model and the critical approach velocity model. The first model is the most common for the homogeneous flow regime. This work suggests that the best model in the case of heterogeneous flow regime is the critical approach velocity model. This work couples the most suitable breakage kernels and the collision frequency with the critical approach velocity model. The Sauter mean diameter of the bubbles is well predicted for both the homogeneous and the heterogeneous flow regimes. The radial profile of the Sauter mean diameter is parabolic in case of heterogeneous flow regime and flat in case of homogeneous flow regime, as observed in the experiments.

Turbulence properties generally influence the coalescence and breakage kernels. Turbulence dissipation rate plays a direct role in the bubble dynamics. Different turbulence models are tested. The effect of the turbulence model choice on the bubble size appears as quite small in the tested operating conditions. It is possible to conclude that the coalescence and breakage kernels studied in this work are usable with different classical turbulence models involved in two-fluid models.

The breakage model of Laakkonen et al. (2006), the modified collision frequency model of Wang et al. (2005b) and the critical approach velocity model for coalescence efficiency of Lehr et al. (2002) seem to be the most relevant with demineralised water at high superficial gas velocities using a homogeneous gas sparger. They have been successfully applied to 
less coalescent systems (ethanol solutions) and also on a bubble column equipped with a different sparger generating big bubbles. Other experiments with different geometries and spargers could be useful to further validate these models. It would be very interesting to do new experiments with larger columns in the future. Contaminants and additives effect can be taken into account with an adjusted critical approach velocity. Globally, a good agreement has been found between CFD-PBM simulations and experimental data. However, to obtain a fully predictive tool that accounts the contamination effects, more experiments with different additives and operating conditions are still necessary. It would be useful to clarify the link between the physical properties of fluids and the coalescence behaviour.

The necessity of considering the velocity field of multiple dispersed gaseous phases could be an important evolution of the method implemented in this work. The drag force closure adopted in this work can predict correctly the hydrodynamics in bubble columns under the heterogeneous flow regime for two-fluid models with a single velocity field for the gaseous phase. The PBM effect on the hydrodynamics is demonstrated to be low with the PBM model adopted in this work. It could be interesting to repeat this sensitivity analysis with a PBM that considers the velocity field of multiple dispersed gaseous phases as bubble size radial segregation might be enhanced. Lift force could have higher effects if a multi-fluid approach was adopted. A comparison on the effect of the lift force between two-fluid approach and multi-fluid approach can be a useful perspective. A comprehensive comparison on the different aspects between the model adopted in this work and the models that consider the velocity field of multiple dispersed gaseous phases would 
be very interesting, in order to highlight advantages and drawbacks.

\section{Notation}

$A_{i} \quad$ Interfacial area concentration, $\mathrm{m}^{-1}$

$\bar{b}_{i}^{k} \quad k^{\text {th }}$ moment of $\beta, \mathrm{m}^{k}$

$c_{1}-c_{5} \quad$ Breakage numerical constants

$c_{1}^{\prime}-c_{3}^{\prime} \quad$ Coalescence numerical constants

$c_{\mathrm{VM}} \quad$ Virtual mass coefficient

$c_{\mu} \quad$ Turbulent viscosity constant

$c_{\Pi} \quad$ Correction term of Wu et al. (1998)

D Column diameter, $m$

$d_{S} \quad$ Diameter of an isosurface spherical bubble, $\mathrm{m}$

$d_{\text {Sauter }}$ Sauter mean diameter, $\mathrm{m}$

$d_{V} \quad$ Diameter of an isovolumic spherical bubble, $\mathrm{m}$

$g \quad$ Breakage kernel, $\mathrm{s}^{-1}$

g Gravity acceleration, $\mathrm{ms}^{-2}$

$h \quad$ Coalescence kernel, $\mathrm{m}^{3} \mathrm{~s}^{-1}$

$\mathrm{H} \quad$ Measurement height, $\mathrm{m}$

$\mathrm{H}_{l} \quad$ Height of liquid into the column, $\mathrm{m}$ 
$h_{0} \quad$ Collision frequency, $\mathrm{m}^{3} \mathrm{~s}^{-1}$

$k \quad$ Turbulence kinetic energy, $\mathrm{m}^{2} \mathrm{~s}^{-2}$

L Bubble size, $\mathrm{m}$

$L_{i} \quad$ Node of the quadrature approximation, $\mathrm{m}$

$m_{k} \quad$ Moment of order $k$ of the BSD, $\mathrm{m}^{k-3}$

$n(L) \quad$ Bubble size distribution, $\mathrm{m}^{-3}$

P Power consumption, W

$p \quad$ External pressure, $\mathrm{Pa}$

$R e_{b} \quad$ Bubble Reynolds number

$S_{12} \quad$ Collision-sectional area, $\mathrm{m}^{2}$

$S_{\varepsilon} \quad$ Source terms for the equation of $\varepsilon, \mathrm{m}^{2} \mathrm{~s}^{-4}$

$S_{k} \quad$ Source terms for the equation of $k, \mathrm{~m}^{2} \mathrm{~s}^{-3}$

$S_{m_{k}} \quad$ Source term for the $k^{\text {th }}$ moment, $\mathrm{m}^{k-3} \mathrm{~s}^{-1}$

$S_{t o t} \quad$ Total surface of the bubbles, $\mathrm{m}^{2}$

$t_{C} \quad$ Contact time, $\mathrm{s}$

$t_{D} \quad$ Drainage time, $\mathrm{s}$

$u_{b} \quad$ Bubble velocity, $\mathrm{m} \mathrm{s}^{-1}$

$u_{\text {crit }} \quad$ Critical approach velocity, $\mathrm{ms}^{-1}$ 
$\mathbf{u}_{\mathrm{g}} \quad$ Gas phase average velocity, $\mathrm{ms}^{-1}$

$u_{\text {rel }} \quad$ Relative approach velocity of the bubbles, $\mathrm{ms}^{-1}$

$u_{s g} \quad$ Superficial gas velocity, $\mathrm{m} \mathrm{s}^{-1}$

V Volume, $\mathrm{m}^{3}$

$V_{t o t} \quad$ Total volume of the bubbles, $\mathrm{m}^{3}$

$w_{i} \quad$ Weight of the quadrature approximation, $\mathrm{m}^{-3}$

$\alpha_{g} \quad$ Gas volume fraction

$\alpha_{\max }$ Gas volume fraction packing limit

$\beta \quad$ Daughter distribution function

$\gamma \quad$ Space-reduction correction factor

$\varepsilon \quad$ Turbulence dissipation rate, $\mathrm{m}^{2} \mathrm{~s}^{-3}$

$\lambda \quad$ Coalescence efficiency

$\mu_{l} \quad$ Liquid phase viscosity, $\mathrm{Pas}$

$\Pi \quad$ Turbulence-length correction factor

$\rho_{g} \quad$ Gas phase density, $\mathrm{kg} \mathrm{m}^{-3}$

$\rho_{l} \quad$ Liquid phase density, $\mathrm{kg} \mathrm{m}^{-3}$

$\sigma \quad$ Surface tension, $\mathrm{N} \mathrm{m}^{-1}$

$\phi \quad$ Column diameter, $\mathrm{m}$

$\omega \quad$ Specific turbulence dissipation rate, $\mathrm{s}^{-1}$ 


\section{References}

Alopaeus, V., Koskinen, J., Keskinen, K. I. and Majander, J. (2002), 'Simulation of the population balances for liquid-liquid systems in a nonideal stirred tank. part 2 - parameter fitting and the use of the multiblock model for dense dispersions', Chemical Engineering Science 57(10), 1815-1825.

Andersson, B., Andersson, R., Håkansson, L., Mortensen, M., Sudiyo, R., Van Wachem, B. and Hellström, L. (2012), Computational Fluid Dynamics for Engineers, first edn, University Press, Cambridge, UK.

Baird, M. H. I. (1992), 'Bubble column reactors. by w.-d. deckwer, wiley, new york, 1991', American Institute of Chemical Engineering Journal 38(8), 1305-1305.

Buffo, A. (2012), Multivariate population balance for turbulent gas-liquid flows., PhD thesis, Politecnico di Torino, Torino, Italy.

Buffo, A., Vanni, M., Marchisio, D. L. and Fox, R. O. (2013), 'Multivariate Quadrature-Based Moments Methods for turbulent polydisperse gas-liquid systems', International Journal of Multiphase Flow 50, 41-57.

Chaumat, H., Billet, A. and Delmas, H. (2007), 'Hydrodynamics and mass transfer in bubble column: Influence of liquid phase surface tension', Chemical Engineering Science 62, 7378-7390.

Chesters, A. K. (1991), 'The modeling of coalescence processes in fluid-liquid dispersions: A review of current understanding', Chemical Engineering 
Research and Design: transactions of the Institution of Chemical Engineers: Part A 69, 259-270.

Colella, D., Vinci, D., Bagatin, R., Masi, M. and Abu Bakr, E. (1999), 'A study on coalescence and breakage mechanisms in three different bubble columns', Chemical Engineering Science 54(21), 4767-4777.

Coulaloglou, C. A. (1975), Dispersed phase interactions in an agitated flow vessel, $\mathrm{PhD}$ thesis, Illinois Institute of Technology, Chicago, Illinois.

Coulaloglou, C. A. and Tavlarides, L. L. (1977), 'Description of interaction processes in agitated liquid-liquid dispersions', Chemical Engineering Science 32, 1289-1297.

Doubliez, L. (1991), 'The drainage and rupture of a non-foaming liquid film formed upon bubble impact with a free surface', International Journal of Multiphase Flow 17, 783-803.

Ekambara, K., Dhotre, M. T. and Joshi, J. B. (2005), 'CFD simulations of bubble column reactors: 1d, 2d and 3d approach', Chemical Engineering Science 60(23), 6733-6746.

Falzone, S., Buffo, A., Vanni, M. and Marchisio, D. L. (2018), 'Simulation of turbulent coalescence and breakage of bubbles and droplets in the presence of surfactants, salts, and contaminants', Advances in Chemical Engineering $52,125-188$.

Friedlander, S. K. (1977), Smoke, Dust and Haze, Wiley, New York. 
Fu, X. Y. and Ishii, M. (2003a), 'Two-group interfacial area transport in vertical air-water flow -II. model evaluation', Nuclear Engineering and Design 219(2), 169-190.

Fu, X. Y. and Ishii, M. (2003b), 'Two-group interfacial area transport in vertical air-water flow: I. mechanistic model', Nuclear Engineering and Design 219(2), $14-168$.

Gemello, L., Cappello, V., Augier, F., Marchisio, D. L. and Plais, C. (2018b), 'CFD-based scale-up of hydrodynamics and mixing in bubble columns', Chemical Engineering Research and Design 136, 846-858.

Gemello, L., Plais, C., Augier, F., Cloupet, A. and Marchisio, D. L. (2018a), 'Hydrodynamics and bubble size in bubble columns: Effects of contaminants and spargers', Chemical Engineering Science 184, 93-102.

Guédon, G. R., Besagni, G. and Inzoli, F. (2017), 'Prediction of gasliquid flow in an annular gap bubble column using a bi-dispersed eulerian model', Chemical Engineering Science 161, 138-150.

Hibiki, T. and Ishii, M. (2000), 'One-group interfacial area transport of bubbly flows in vertical round tubes', International Journal of Heat and Mass Transfer 43, 2711-2726.

Hibiki, T., Takasama, T. and Ishii, M. (2001), 'Interfacial area transport of bubbly flow in a small diameter pipe', Journal of Nuclear Science and Technology 38, 614-620.

Hillmer, G., Weismantel, L. and Hofmann, H. (1994), 'Investigations 
and modelling of slurry bubble columns', Chemical Engineering Science $49(6), 837-843$.

Howarth, W. J. (1964), 'Coalescence of drops in a turbulent flow field', Chemical Engineering Science 19, 33-38.

Jakobsen, H. A., Lindborg, H. and Dorao, C. A. (2005), 'Modeling of bubble column reactors: Progress and limitations', Industrial \&6 Engineering Chemistry Research 44(14), 5107-5151.

Jeffreys, G. V. and Davies, G. A. (1971), Coalescence of Liquid Droplets and Liquid Dispersion: Recent Advances in Liquid-Liquid Extraction, 1 st edn, Pergamon Press, Oxford, UK.

Joshi, J. B. (2001), 'Computational flow modelling and design of bubble column reactors', Chemical Engineering Science 56(21), 5893-5933.

Kalkach-Navarro, S., Lahey Jr., R. T. and Drew, D. A. (1994), 'Analysis of the bubbly/slug flow regime transition', Nuclear Engineering and Design 151(1), 15-39.

Kataoka, I., Besnard, D. C. and Serizawa, A. (1992), 'Basic equation of turbulence and modelling of interfacial transfer terms in gas-liquid twophase flow', Chemical Engineering Communications 118, 221-236.

Kennard, E. H. (1938), Kinetic Theory of Gases, McGraw-Hill, New York.

Krepper, E., Lucas, D., Frank, T., Prasser, H.-M. and J. Zwart, P. (2008), 'The inhomogeneous MUSIG model for the simulation of polydispersed flows', Nuclear Engineering and Design 238, 1690-1702. 
Kuboi, R., Komasawa, I. and Otake, T. (1972), 'Collision and coalescence of dispersed drops in turbulent liquid flow', Journal of Chemical Engineering of Japan 5, 423-424.

Laakkonen, M., Alopaeus, V. and Aittamaa, J. (2006), 'Validation of bubble breakage, coalescence and mass transfer models for gas-liquid dispersion in agitated vessel', Chemical Engineering Science 61, 218-228.

Laakkonen, M., Moilanen, P., Alopaeus, V. and Aittamaa, J. (2007), 'Modelling local bubble size distributions in agitated vessels', Chemical Engineering Science 62, 721-740.

Lee, C. H., Erickson, L. E. and Glasgow, L. A. (1987), 'Bubble break-up and coalescence in turbulent gas-liquid dispersions', Chemical Engineering Communications 59, 65-84.

Lehr, F. and Mewes, D. (1999), 'A transport equation for the interfacial area density applied to bubble columns', Chemical Engineering Science $56,1159-1166$.

Lehr, F., Millies, M. and Mewes, D. (2002), 'Bubble-size distributions and flow fields in bubble columns', American Institute of Chemical Engineering Journal 48(11), 2426-2443.

Liao, Y. and Lucas, D. (2009), 'A literature review of theoretical models for drop and bubble breakup in turbulent dispersions', Chemical Engineering Science 64, 3389-3406.

Liao, Y. and Lucas, D. (2010), 'A literature review on mechanisms and 
models for the coalescence process of fluid particles', Chemical Engineering Science 65, 2851-2864.

Lucas, D. and Tomiyama, A. (2011), 'On the role of the lateral lift force in poly-dispersed bubbly flows', International Journal of Multiphase Flow 37, 1178-1190.

Luo, H. (1993), Coalescence, breakup and liquid circulation in bubble column reactors, PhD thesis, The Norwegian Institute of Technology, Trondheim, Norway.

Luo, H. and Svendsen, H. F. (1996), 'Modeling and simulation of binary approach by energy conservation analysis', Chemical Engineering Communications 145, 145-153.

Marchisio, D. L. and Fox, R. O. (2013), Computational Models for Polydisperse Particulate and Multiphase Systems, Cambridge Series in Chemical Engineering, Cambridge University Press, Cambridge, UK.

Martínez-Bazán, C., Montañés, J. L. and Lasheras, J. C. (1999), 'On the breakup of an air bubble injected into a fully developed turbulent flow. part 1. breakup frequency', Journal of Fluid Mechanics 401, 157-182.

McClure, D. D., Kavanagh, J. M., Fletcher, D. F. and Barton, G. W. (2013), 'Development of a CFD model of bubble column bioreactors: Part one - a detailed experimental study', Chemical Engineering $\&$ Technology 36(12), 2065-2070.

McClure, D. D., Kavanagh, J. M., Fletcher, D. F. and Barton, G. W. (2017), 
'Experimental investigation into the drag volume fraction correction term for gas-liquid bubbly flows', Chemical Engineering Science 170, 91-97.

McClure, D. D., Norris, H., Kavanagh, J. M., Fletcher, D. F. and Barton, G. W. (2015), 'Towards a CFD model of bubble columns containing significant surfactant levels', Chemical Engineering Science 127, 189-201.

McClure, D. D., Wang, C., Kavanagh, J. M., Fletcher, D. F. and Barton, G. W. (2016), 'Experimental investigation into the impact of sparger design on bubble columns at high superficial velocities', Chemical Engineering Research and Design 106, 205-213.

Menzel, T., In der Weide, T., Staudacher, O., Wein, O. and Onken, U. (1990), 'Reynolds stress model for bubble column reactor', Industrial $\mathscr{G}$ Engineering Chemistry Research 29(6), 988-994.

Narasimhan, G., Ramakrishna, D. and Gupta, J. P. (1979), 'A model for transitional breakage probability of droplets in agitated lean liquid-liquid dispersions', Chemical Engineering Science 34, 257-265.

Park, J. Y. and Blair, L. M. (1975), 'The effect of coalescence on drop size distribution in an agitated liquid-liquid dispersion', Chemical Engineering Science 30, 1057-1064.

Petitti, M., Nasuti, A., Marchisio, D. L., Vanni, M., Baldi, G., Mancini, N. and Podenzani, F. (2010), 'Bubble size distribution modeling in stirred gasliquid reactors with QMOM augmented by a new correction algorithm.', American Institute of Chemical Engineering Journal 56, 36-53. 
Prince, M. J. and Blanch, H. W. (1990), 'Bubble coalescence and break-up in air-sparged bubble columns', American Institute of Chemical Engineering Journal 36, 1485-1499.

Raimundo, P. M. (2015), Analysis and modelization of local hydrodynamics in bubble columns, $\mathrm{PhD}$ thesis, Université Grenoble Alpes.

Raimundo, P. M., Cartellier, A., Beneventi, D., Forret, A. and Augier, F. (2016), 'A new technique for in-situ measurements of bubble characteristics in bubble columns operated in the heterogeneous regime', Chemical Engineering Science 155, $504-523$.

Raimundo, P. M., Cloupet, A., Cartellier, A., Beneventi, D. and Augier, F. (2019), 'Hydrodynamics and scale-up of bubble columns in the heterogeneous regime: Comparison of bubble size, gas holdup and liquid velocity measured in 4 bubble columns from $0.15 \mathrm{~m}$ to $3 \mathrm{~m}$ in diameter', Chemical Engineering Science 198, 52 - 61.

Ranade, V. (1997), 'Modelling of turbulent flow in a bubble column reactor', Chemical Engineering Research and Design 75(1), 1- 23. Process and Product Development.

Ranade, V. V. (2002), Computational Flow Modeling for Chemical Reactor Engineering, Academic Press, San Diego, California, USA.

Renardy, Y., Cristini, V. and Li, J. (2002), 'Drop fragment distributions under shear with inertia', International Journal of Multiphase Flow $28,1125-1147$. 
Ribeiro, Jr, C. P. and Mewes, D. (2007), 'The influence of electrolytes on gas hold-up and regime transition in bubble columns', Chemical Engineering Science 62(17), $4501-4509$.

Roels, J. A. and Heijnen, J. J. (1980), 'Power dissipation and heat production in bubble columns: Approach based on nonequilibrium thermodynamics', Biotechnology and Bioengineering 22(11), 2399-2404.

Sánchez Pérez, J. A., Rodríguez Porcel, E. M., Casas López, J., FernandezSevilla, J. M. and Chisti, Y. (2006), 'Shear rate in stirred tank and bubble column bioreactors', Chemical Engineering Journal 124, 1-5.

Sato, Y. and Sekoguchi, K. (1975), 'Liquid velocity distribution in two-phase bubble flow', International Journal of Multiphase Flow 2(1), 79-95.

Shinnar, R. and Church, J. M. (1960), 'Predicting particle size in agitated dispersions', Industrial and Engineering Chemistry 52, 253-256.

Simon, M. (2004), Koaleszenz von Tropfen und Tropfenschwärmen, PhD thesis, Teschinschen Universtität Kaiserslautern, Kaiserslautern, Germany.

Simonin, O. and Viollet, P. L. (1990), 'Modeling of turbulent two-phase jets loaded with discrete particles', Phenomena in Multiphase Flows pp. 259269.

Simonnet, M., Centric, C., Olmos, E. and Midoux, N. (2008), 'CFD simulation of the flow field in a bubble column reactor: Importance of the drag force formulation to describe regime transitions.', Chemical Engineering and Processing 47, 1726-1737. 
Sovova, H. (1981), 'Breakage and coalescence of drops in a batch stirred vessel - ii comparison of model and experiments', Chemical Engineering Science $36,1567-1573$.

Tabib, M. V., Roy, S. A. and Joshi, J. B. (2008), 'CFD simulation of bubble column - An analysis of interphase forces and turbulence models', Chemical Engineering Journal 139(3), 589-614.

Tisnadjaja, D., Gutierrez, N. A. and Maddox, I. S. (1996), 'Citric acid production in a bubble-column reactor using cells of the yeast Candida guilliermondii immobilized by adsorption onto sawdust', Enzyme and Microbial Technology 19(5), 343-347.

Tomiyama, A. (1998), 'Struggle with computational bubble dynamics.', Multiphase Science and Technology 10(4), 369.

Troshko, A. (2006), 'Best practice for modelling bubble column reactors with FLUENT.', ANSYS presentation.

Troshko, A. A. and Hassan, Y. A. (2001), 'A two-equation turbulence model of turbulent bubbly flows', International Journal of Multiphase Flow 27(11), 1965-2000.

Wang, T. F., Wang, J. F. and Jin, Y. (2003), 'A novel theoretical breakup kernel function for bubbles/droplets in a turbulent flow', Chemical Engineering Science 58(20), 4629-4637.

Wang, T. F., Wang, J. F. and Jin, Y. (2005a), 'Population balance model for gas-liquid flows: Influence of bubble coalescence and breakup models', Industrial \& Engineering Chemistry Research 44(11), 7540-7549. 
Wang, T. F., Wang, J. F. and Jin, Y. (2005b), 'Theoretical prediction of flow regime transition in bubble columns by the population balance model', Chemical Engineering Science 60, 6199-6209.

Wu, Q., Kim, S. and Ishii, M. (1998), 'One-group interfacial area transport in vertical bubbly flow', International Journal of Heat and Mass Transfer 41, 1103-1112.

Xue, J. (2004), Bubble velocity, size and interfacial area measurements in bubble columns, PhD thesis, Sever Institute of Washington University, St. Louis, Missouri.

Yao, W. and Morel, C. (2004), 'Volumetric interfacial area prediction in upward bubbly two-phase flow', International Journal of Heat and Mass Transfer 47, 307-328. 